\title{
Temperature Overloads in Power Grids Under Uncertainty: A Large Deviations Approach
}

\author{
Tommaso Nesti ${ }^{\circledR}$, Jayakrishnan Nair $\left.{ }^{(}\right)$, and Bert Zwart, Member, IEEE
}

\begin{abstract}
The advent of renewable energy has huge implications for the design and control of power grids. Due to increasing supply-side uncertainty, traditional reliability constraints, such as strict bounds on current, voltage, and temperature in a transmission line have to be replaced by computationally demanding chance constraints. In this paper, we use large deviation techniques to study the probability of current and temperature overloads in power grids with stochastic power injections, and develop corresponding safe capacity regions. In particular, we characterize the set of admissible power injections such that the probability of overloading of any line over a given time interval stays below a fixed target. We show how enforcing (stochastic) constraints on temperature, rather than on current, results in a less conservative approach and can thus lead to capacity gains.
\end{abstract}

Index Terms-Chance constraints, energy systems, large deviations (LD) theory, network analysis and control, optimal power flow (OPF), temperature overload, uncertainty.

\section{INTRODUCTION}

$\mathbf{T}$ HE electricity network is one of the backbones of modern society, and is expected to function at all times. The advent of renewable energy sources, such as wind and solar generation, has put this requirement under pressure due to their considerable intermittency. Both the U.S. and Europe have set long-term goals on the usage of renewable energy, but the effects of the integration of renewable sources into the power grid are already felt today. For example, $80 \%$ of the bottlenecks in the European transmission grid are already caused by renewables [1]. Dealing with the uncertainty of renewable generation effectively is therefore an essential requirement in the operation of modern grids.

A well-controlled power grid matches supply and demand at all times, ensuring that line constraints are not violated. The system operator achieves this by making periodic control actions

Manuscript received December 24, 2018; revised May 17, 2019 and May 22, 2019; accepted May 30, 2019. Date of publication June 12, 2019; date of current version September 17, 2019. This work was supported by the NWO VICl Grant 639.033.413. Recommended by Associate Editor M. Chertkov. (Corresponding author: Tommaso Nesti.)

T. Nesti is with the Centrum Wiskunde \& Informatica, Amsterdam 1098 XG, The Netherlands (e-mail: nesti@ cwi.nl).

J. Nair is with the Indian Institute of Technology Bombay, Mumbai 400076 India (e-mail: jayakrishnan.nair@ee.iitb.ac.in).

B. Zwart is with the Centrum Wiskunde \& Informatica, Amsterdam 1098 XG, The Netherlands, and also with the Eindhoven University of Technology, Eindhoven 5612 AZ, The Netherlands (e-mail: bert.zwart@cwi.nl).

Digital Object Identifier 10.1109/TCNS.2019.2922492 (typically every 5-15 $\mathrm{min}$ ) that adapt the operating point of the grid in response to changing conditions [2]. A key assumption driving grid operation today is that the grid remains roughly static between control instants. In other words, it is assumed that the operating point does not change much between control instants. Thus, the operator simply ensures that line constraints are satisfied at each control instant. This assumption is of course reasonable when there is little short-term uncertainty in demand and supply.

However, with increasing penetration of renewable sources, supply-side uncertainty is bound to grow dramatically going forward. Renewable energy sources, such as wind and solar, can exhibit considerable variability in power generation in the short term [3], [4]. Consequently, in the near future, system operators will no longer be able to assume that the grid is static between control instants, and will have to set the operating point taking into account its variability in the short term. This entails setting the operating point of the grid with stochastic guarantees on constraint satisfaction [5], [6]. In other words, the operating point must be set such that line constraint violation is a sufficiently rare event until the next control instant. Moreover, schemes, such as optimal power flow (OPF), need to be adapted in such a way that uncertainty is taken into account, and outages stay rare events.

In an optimization framework, this leads to chance constraints that are hard to evaluate analytically. The analysis of such constraints, such as the probability of overheating or a blackout, is often done using rare event simulation techniques [6], [7]. Although detailed simulations can be more accurate, short-term planning requires tools that enable the grid operator to handle the stochastic constraints much faster.

The main contribution of this paper is the development of tractable capacity regions for a power grid with variable sources. Specifically, we characterize the set of admissible power injections such that the overheating of any transmission line over a given interval is a rare event. For the simplest network with two nodes and one line, our results have been published without proof in the extended abstract [8].

Our main technique to achieve this is the theory of large deviations (LD). Specifically, we model the random power input sources as small-noise stochastic differential equations (SDE), for which a comprehensive and sufficiently explicit theory of $\mathrm{LD}$ is available. SDEs are a flexible modeling tool for continuously varying processes, and their use for wind speed modeling has been adopted recently by several authors [9]-[12]. We model power flows on the network using the dc approximation, which 
is standard in the literature of high-voltage transmission system analysis [13]-[15]. More realistic models based on ac power flow are often analytically intractable and may not even be well posed [16]-[18]. This allows us to apply Freidlin-Wentzell theory [19] to approximate the probability of an overload event, which, in turn, leads to our capacity region characterization.

Avoiding transmission line overheating is a key reliability constraint in order to avoid sag and loss of tensile strength [20], one of the key causes of the Northeast blackout in 2003 [21] and the San Diego blackout in 2011 [22]. The classical approach for enforcing this constraint is to impose a certain upper bound on each line current. In Section III, we follow this approach and develop capacity regions based on bounding the probability that any line current exceeds its limit over a given interval. We prove an important convexity property of this capacity region, which enables its application in optimization formulations, such as OPF. When the random power injections are modeled by an Ornstein-Uhlenbeck (OU) process, we express this capacity region in closed form.

Since line temperature responds gradually to current, transient current overload does not necessarily imply a temperature overload. Thus, imposing a constraint on the probability of current overload results in a smaller capacity region compared to the same constraint on the probability of temperature overload. This observation was noted via simulations in [6]. We show that it is possible to develop LD estimates for temperature constraint violations that lead to larger capacity regions, than the ones obtained when only considering currents. To the best of our knowledge, this paper provides the first analytical treatment of this phenomenon.

As it turns out, it is hard to compute such regions. We overcome this issue by developing two tractable approximations: the first is an inner bound, and the second is based on a Taylor series expansion of the decay rate of the temperature overload probability. Both of the two regions coming out of these approximations capture the benefits of incorporating the transient relationship between temperature and current. Moreover, they both have the same computational complexity as the currentbased capacity region. For the case of OU power injections, we express these capacity regions in closed form.

There are several related strands of the literature, apart from the papers dealing with rare event simulation that have been mentioned earlier. Much of the literature on power flow in electricity grids considers deterministic settings, focusing on computational and/or optimization issues. Power flow papers that analyze stochastic models include [5], [20], [23], and [24].

One remark about these papers is that they model stochastic behavior at particular snapshots of time, as opposed to the process-level model in this paper. Process-level models have been considered in simulation studies [6] and in recent works on chance-constrained versions of OPF [25], [26]. Other papers on chance-constrained OPFs include [27], where the authors integrate probabilistic guarantees in a dc OPF via a scenario approach, and recent works on chance-constrained ac OPF, which tackle the nonlinearities by means of convex approximations [28], relaxations [29], and local linearization around a forecasted operating point [30].
In this paper, we propose to approximate chance-constraints by using LD theory. This is markedly different from the approach of using Monte Carlo methods [31]. Such methods can be effective but can require a large number of samples. Specifically, they are difficult to implement in our dynamic continuous-time setting. Another approach is to develop analytic convex approximations along the lines suggested in [32]. Unfortunately, this method also seems mainly designed for static problems, and it appears hard to implement it in our dynamic setting.

In this paper, we aim to develop analytic tools that are explicit enough so as to be useful for planning and control of power grids in the short term. This paper is complementary to recent efforts on managing supply-side uncertainty via demand response [33], [34], energy storage [35], [36], and market (re)design [37], [38]. A recent work on the analysis of temperature constraints in a discrete-time setting is [39].

This paper is organized as follows. In Section II, we describe our model for power injections, line currents, and line temperatures. Sections III and IV constitute the core of this paper: we develop and characterize LD-based capacity regions for line currents and line temperatures, respectively, and provide explicit expressions in the particular case that the power injections follow a multivariate OU process. In Section $\mathrm{V}$, numerics for the OU case are presented. We summarize and discuss future directions in Section VI. Proofs are reported in the appendix.

\section{SYSTEM MODEL}

\section{A. Model for the Power Grid and DC Approximation}

The network is specified by a connected graph $\mathcal{G}=(\mathcal{N}, \mathcal{L})$, where $\mathcal{N}=\{0,1,2, \ldots, N\}$ is the set of nodes, modeling buses, and $\mathcal{L}$ is the set of edges, representing the transmission lines. We have $|\mathcal{N}|=N+1$ and $|\mathcal{L}|=L$. After choosing an arbitrary but fixed orientation of the transmission lines, we denote by $\ell=(i, j) \in \mathcal{L}$ the transmission line between buses $i$ and $j$, and by $w_{\ell}=w_{i, j}=w_{j, i}>0$ the weight of edge $\ell=(i, j)$, corresponding to the susceptance of that transmission line. By convention, if there is no line between $i$ and $j$, we set $w_{i, j}=w_{j, i}=0$. The network structure is described by the $e d g e-$ vertex incidence matrix $A \in \mathbb{R}^{L \times(N+1)}$ defined as

$$
A_{\ell, i}= \begin{cases}1, & \text { if } \ell=(i, j) \\ -1, & \text { if } \ell=(j, i) . \\ 0, & \text { otherwise }\end{cases}
$$

Denote by $W$ the $L \times L$ diagonal matrix defined as $W:=$ $\operatorname{diag}\left(w_{1}, \ldots, w_{L}\right)$. The network topology and weights are encoded in the weighted Laplacian matrix of the graph $G$, defined as $B:=A^{\top} W A$ or entrywise as

$$
B_{i, j}=\left\{\begin{array}{ll}
-w_{i, j}, & \text { if } i \neq j \\
\sum_{k \neq i} w_{i, k}, & \text { if } i=j
\end{array} .\right.
$$

Let $S(t)=\left(S_{i}(t)\right)_{i \in \mathcal{N}}$ denote the vector of active net power injections at time $t$, with the convention that $S_{i}(t) \geq 0\left(S_{i}(t) \leq\right.$ $0)$ means that power is generated (consumed, respectively) at 
bus $i$. Node 0 models the slack bus, which ensures that there are no active power imbalances in the network.

Let $I(t)=\left(I_{\ell}(t)\right)_{\ell \in \mathcal{L}}$ be the vector of line currents, and $K(t)=\left(K_{\ell}(t)\right)_{\ell \in \mathcal{L}}$ be the vector of line temperatures. Each transmission line $\ell$ is associated with a thermal limit $K_{\ell, \max }$, which is the maximum permissible temperature of the line [20]. We define $I_{\ell, \max }>0$ such that if $\left|I_{\ell}(t)\right|=I_{\ell, \max }$ at all times, then $\lim _{t \rightarrow \infty} K_{\ell}(t)=K_{\ell \text {,max }}$. Throughout this paper, we work with normalized currents $Y(t)=\left(Y_{\ell}(t)\right)_{\ell \in \mathcal{L}}$, defined as $Y_{\ell}(t)=I_{\ell}(t) / I_{\ell, \max }$.

In order to model the relation between power injections and line currents, we make use of the $d c$ approximation (a.k.a. DC power flow, [13]), which leads to a linear relationship between power injections and normalized line currents of the form $Y(t)=\bar{C} S(t)$, where $\bar{C} \in \mathbb{R}^{L \times(N+1)}$ is a matrix encoding the network topology and weights [see (4)].

In the remainder of this section, we briefly recall the dc approximation. For notational simplicity, we suppress the dependence of power, voltage, and current on time when not essential. Let $V_{j}=\left|V_{j}\right| e^{\mathrm{i} \theta_{j}}$ denote the voltage at node $j$, with $V_{j}$ the voltage magnitude and $\theta_{j}$ the voltage phase. The dc approximation consists of the following three assumptions.

i) Voltage magnitudes $\left|V_{j}\right|$ are all equal to 1 (in the per-unit system)

ii) The phase differences between neighboring nodes are small: $\forall(i, j) \in \mathcal{L},\left|\theta_{i}-\theta_{j}\right| \ll 1$.

iii) The resistances of transmission lines are negligible with respect to the reactances.

Under these assumptions, the reactive power flows are negligible compared to the active power flows. Moreover, the active power flow $F_{i, j}$ on line $(i, j) \in \mathcal{L}$ can be approximated as $F_{i, j} \approx w_{i, j}\left(\theta_{i}-\theta_{j}\right)$. This allows us to express the vector of (active) node power injections $S \in \mathbb{R}^{N+1}$ in matrix form as

$$
S=B \theta
$$

where $\theta \in \mathbb{R}^{N+1}$ is the vector of phase angles. Note that $\sum_{i \in \mathcal{N}} S_{i}=0$, which is consistent with above-mentioned Assumption (iii) ignoring line losses. Under the dc approximation, one can also approximate the line currents with the (active) power flow on the line $I_{i, j} \approx w_{i, j}\left(\theta_{i}-\theta_{j}\right)$. The aforementioned equivalence between the currents and power flows under this approximation has been noted before (see, for example, [40]). Expressing the vector of line currents in matrix form, we obtain

$$
I=W A \theta .
$$

Following the derivation in [26], without loss of generality, we can set the phase angle at the slack bus equal to zero, i.e., $\theta_{0}=0$, and rewrite the system $S=B \theta$ as

$$
\theta=\breve{B} S
$$

where $\breve{B}=\left(\begin{array}{cc}0 & 0 \\ 0 & \hat{B}^{-1}\end{array}\right)$ and $\hat{B}$ is the $N \times N$ submatrix obtained from $B$ by deleting the first row and first column.

Recall that the normalized currents are defined as $Y_{\ell}(t)=$ $I_{\ell}(t) / I_{\ell, \max }$, and let $\Lambda=\operatorname{diag}\left(1 / I_{1, \max }, \ldots, 1 / I_{L, \max }\right)$. In view of (2) and (3), the active normalized line currents $Y$ can be written as a linear transformation of the power injections $S$, i.e., $Y(t)=\bar{C} S(t)$, where $\bar{C}:=\Lambda W A \breve{B}$.

\section{B. Stochastic and Deterministic Power Injections}

We assume that power injections at nodes $1, \ldots, m \leq N$ are stochastic, modeling buses housing intermittent renewable power generation. On the other hand, power injections at nodes $m+1, \ldots, N$ are assumed to be deterministic and constant, modeling conventional loads/generators.

We will be interested in capturing the probability of current/temperature overload over a finite horizon $[0, T]$, which corresponds to the interval between periodic control actions by the grid operator. Thus, the buses in $\{m+1, \ldots, N\}$ are those that may be assumed to have a steady power injection over this time scale, denoted by $\mu_{D}$. Note that the power injection at the slack node 0 is also stochastic, since $S_{0}(t)=-\sum_{i=1}^{n} S_{i}(t)$. The power injection vector is of the form $S(t)=\left(S_{0}(t), X(t), \mu_{D}\right)$, where $X(t) \in \mathbb{R}^{m}$ is the vector of stochastic injections, and $\mu_{D}=\left(\mu_{D, i}\right)_{i=m+1}^{N} \in \mathbb{R}^{N-m}$. We denote the initial condition for the stochastic power injections by $\mu:=X(0)$, and let $\bar{\mu}:=\left(\mu, \mu_{D}\right)$.

In order to make the dependence of the normalized current on stochastic and deterministic power injections more explicit, we note that we can write

$$
Y(t)=\bar{C} S(t)=\left[\mathbf{0}|C| C_{D}\right]\left[\begin{array}{c}
S_{0}(t) \\
X(t) \\
\mu_{D}
\end{array}\right]
$$

where $\quad \mathbf{0}=[0, \ldots, 0]^{\top} \in \mathbb{R}^{L}, \quad$ and $\quad C \in \mathbb{R}^{L \times m}$ and $C_{D} \in$ $\mathbb{R}^{L \times(n-m)}$ are the submatrices of $\bar{C}$ corresponding to stochastic and deterministic injections, respectively. More compactly

$$
Y(t)=C X(t)+y
$$

where $y:=C_{D} \mu_{D}$. We will refer to (5) as the dc power flow equations. The following lemma shows that matrix $C$ has rank $m$, i.e., the number of stochastic power injections.

Lemma II.1: If the network graph is connected, rank $(\bar{C})=$ $N$ and rank $(C)=m$. In particular, the matrix $C$ has linearly independent columns.

We may interpret $\bar{\mu}=\left(\mu, \mu_{D}\right)$ as the vector of power injections set by the grid operator at time 0 (for example, $\bar{\mu}$ could be the result of an OPF planning). Recall that the initial condition for the normalized currents is $Y(0)=\nu$, where $\nu:=C \mu+y$. We are interested in scenarios where power grids operate safely, by assuming that the nominal power injections $\bar{\mu}$ are such that the corresponding expected line currents at time $t=0$ do not exceed the critical level, i.e., $\|\nu\|_{\infty}=\max _{\ell=1, \ldots, L}\left|\nu_{\ell}\right| \leq 1$ (possibly, several $\left|\nu_{\ell}\right|$ could be close or equal to their threshold, modeling a high-stress situation). Subsequently, some of the power injections fluctuate randomly because of the variability of the renewable generators. Our focus is to characterize the set of power injection vectors $\bar{\mu}$ such that the probability of current/temperature overload over a finite horizon $[0, T]$ is below a prescribed target $p$.

\section{Mapping Between Line Current and Line Temperature}

In this section, we describe how line temperature depends on line current. Recall that $K_{\ell}(t)$ denotes the temperature of line $\ell$. We work with normalized line temperatures, defined as follows. 
Let $K_{\text {env, } \ell}$ be the ambient temperature around line $\ell$. We define the normalized line temperatures $\Theta(t)=\left(\Theta_{\ell}(t)\right)_{\ell \in \mathcal{L}}$ as $\Theta_{\ell}(t)=$ $\frac{K_{\ell}(t)-K_{\text {env }, \ell}}{K_{\max , \ell}-K_{\text {env }, \ell}}$. Note that the reliability constraint on line temperatures reads $\left\|\Theta_{\ell}\right\|_{\infty} \leq 1$, where $\|f\|_{\infty}:=\max _{t \in[0, T]}\|f(t)\|_{\infty}$ for a continuous function $f:[0, T] \rightarrow \mathbb{R}^{L}$.

In this spirit, in Section IV, we characterize the capacity region of the power grid based on bounding the temperature overload probability. In other words, we describe the set of initial power injection vectors $\bar{\mu}$ such that $\mathbb{P}\left(\|\Theta\|_{\infty} \geq 1\right) \leq p$, where $p$ is a prescribed reliability target.

The transient relationship between the normalized temperature $\Theta_{\ell}$ and the normalized current is given by the ordinary differential equation [41]

$$
\tau_{\ell} \frac{d \Theta_{\ell}}{d t}+\Theta_{\ell}=\left(Y_{\ell}\right)^{2}
$$

where $\tau_{\ell}>0$ denotes the thermal constant of the transmission line $l$. Thus, we have

$$
\Theta_{\ell}(t)=\Theta_{\ell}(0) e^{-t / \tau_{\ell}}+\frac{1}{\tau_{\ell}} \int_{0}^{t} e^{-(t-s) / \tau_{\ell}}\left(Y_{\ell}(s)\right)^{2} d s .
$$

Note that the instantaneous line temperature depends on the history of the line current process, with an exponentially decaying weight on past values. The parameter $\tau_{\ell}$ determines the dependence of the instantaneous temperature on past values of current. If $\tau_{\ell}$ is small, the dependence on past current values becomes weaker, i.e., the line temperature responds more quickly to changes in current. In the limit as $\tau_{\ell} \downarrow 0$, the response is instantaneous, i.e., $\Theta_{\ell}(t)=\left(Y_{\ell}(t)\right)^{2}$.

For simplicity, we assume that the initial condition $\Theta_{\ell}(0)=$ $\left(Y_{\ell}(0)\right)^{2}=\nu_{\ell}^{2} \quad \forall \ell \in \mathcal{L}$ for line temperatures. Note that $\nu_{\ell}^{2}$ is the steady-state temperature corresponding to a constant line current $\nu_{\ell} .{ }^{1}$ With the aforementioned initial condition, let us denote the mapping from the current process $Y$ to the temperature process $\Theta$ as $\Theta=\xi_{\tau}(Y)$, where we emphasize the dependence on the thermal time constants $\tau=\left(\tau_{l}\right)_{l \in \mathcal{L}}$.

\section{Stochastic Model for Power Injections}

We now describe our stochastic model for the power injections $X(t)$. Recall that in order to characterize the capacity region of the power grid, we have to estimate the following overload probabilities:

$$
\mathbb{P}\left(\|Y\|_{\infty} \geq 1\right), \quad \mathbb{P}\left(\|\Theta\|_{\infty} \geq 1\right) .
$$

We use the theory of LD to estimate these probabilities. Formally, we model the vector of random power injections $X^{\epsilon}(t)=\left(X_{1}^{\epsilon}(t), \ldots, X_{m}^{\epsilon}(t)\right)$ as the strong solution of the $m$ dimensional SDE

$$
d X^{\epsilon}(t)=b\left(X^{\epsilon}(t)\right) d t+\sqrt{\epsilon} L\left(X^{\epsilon}(t)\right) d W(t)
$$

\footnotetext{
${ }^{1}$ This assumption, which ignores the history of the temperature process prior to time $\approx 0$, is a natural engineering assumption if the actual line temperatures at time $\approx 0$ are unavailable. If such measurements are available, it is possible to incorporate these into our capacity region based on temperature overload (see Section IV-A) as well as its inner bound (see Section IV-B), although the analysis gets more complicated (see [9, Sec. 4.3]).
}

where $X^{\epsilon}(0)=\mu, \quad b(x)=\left(b_{1}\left(x_{1}\right), \ldots, b_{m}\left(x_{m}\right)\right), \quad L(x)=$ $\operatorname{diag}\left(\left\{l_{i}\left(x_{i}\right)\right\}_{i=1, \ldots, m}\right)$, and $W(t)=\left(W_{i}(t)\right)_{i=1, \ldots m}$. The function $b$ is referred to as the drift function and captures the evolution of the process in the absence of noise. The noise in the evolution of the process is introduced by the second term in (8): $W_{i}(t)$ is a standard Brownian motion in $\mathbb{R}$. This noise is modulated in a state-dependent fashion by the diffusion function $L$, and the scaling parameter $\epsilon>0$ captures the amount of randomness in the power injections. As $\epsilon \rightarrow 0$, the magnitude of the noise injected into the evolution of the process $X^{\epsilon}(t)$ diminishes, making LD from the "noise-less" behavior exponentially (in $1 / \epsilon$ ) unlikely.

It is in this regime that LD theory gives us tractable approximations of the probabilities of the rare events corresponding to current and temperature overloads. In practice, $\epsilon$ can be chosen so that the variance of the process $X^{\epsilon}(t)$ matches the estimation error for renewable production over a specific time unit (see Section V-B). We make the following regularity assumptions: $\forall i=1, \ldots, m, b_{i}: \mathbb{R} \rightarrow \mathbb{R}$ is Lipschitz continuous and differentiable with $b_{i}\left(\mu_{i}\right)=0 ; l_{i}: \mathbb{R} \rightarrow(0, \infty)$ is Lipschitz continuous, bounded, and differentiable.

The $\epsilon$-scaled current process $Y^{\epsilon}(t)=\left(Y^{\epsilon}\right)_{\ell \in \mathcal{L}}$ is defined as per the dc power flow equations: $Y^{\epsilon}(t)=C X^{\epsilon}(t)+y$. Similarly, the $\epsilon$-scaled temperature process $\Theta_{\tau}^{\epsilon}(t)=\left(\Theta_{\tau}^{\epsilon}\right)_{\ell \in \mathcal{L}}$, with thermal constant $\tau$, is defined as $\Theta_{\tau}^{\epsilon}=\xi_{\tau}\left(Y^{\epsilon}\right)$. In the following sections, we apply the theory of LD to estimate the probabilities $\mathbb{P}\left(\left\|Y^{\epsilon}\right\|_{\infty} \geq 1\right)$ and $\mathbb{P}\left(\left\|\Theta^{\epsilon}\right\|_{\infty} \geq 1\right)$, in the limit as $\epsilon \downarrow 0$.

\section{Capacity Regions Characterization Based on CURRENT OVERLOAD}

The traditional approach for ensuring line reliability is to impose the condition $\|Y(t)\|_{\infty}:=\max _{\ell \in \mathcal{L}}\left|Y_{\ell}(t)\right| \leq 1$ at all times. In this spirit, in this section, we characterize the capacity region of the power grid obtained by bounding the probability of current overload over $[0, T]$ by a prescribed target $q$

$$
\mathbb{P}\left(\|Y\|_{\infty} \geq 1\right) \leq q .
$$

Our focus is to characterize the space of initial power injections that can be "set" at time 0 , such that the probability that the inherent variability in the stochastic sources leads to a current overload before the next control instant is small. ${ }^{2}$

The aforementioned approach is in line with the conventional technique of enforcing the thermal limits of transmission lines by capping the peak current on each line. In Section IV-A, a more refined approach, taking into account the transient relationship between line current and line temperature, is presented.

In the following, we first provide an LD principle for the current overflow event $\left\{\left\|Y^{\epsilon}\right\| \geq 1\right\}$ in the limit as $\epsilon \downarrow 0$. Next, we use this characterization to define the current-overload-based capacity region, and prove a convexity result that facilitates its application as a constraint in OPF. We then provide two lemmas that are useful for computing the capacity region in practice and we give a closed-form characterization of the capacity region when the stochastic injections follow an OU process.

\footnotetext{
${ }^{2}$ Given the equivalence between line currents and power flows under the $\mathrm{dc}$ approximation, the results in this section can also be interpreted in terms of the probability of exceeding line power flow limits.
} 


\section{A. LD Results}

The theory of LD is concerned with calculating the exponential decay of rare events probabilities, by means of the so-called rate functions. The main idea behind the theory is to provide a rigorous mathematical foundation to the approximation

$$
\mathbb{P}_{\epsilon}(E)=\int_{x \in E} f_{\epsilon}(x) d x \approx \max _{x \in E} f_{\epsilon}(x)
$$

in the regime where $\epsilon$ is small. It turns out that the density $f_{\epsilon}(x)$ (where $x$ can be a function, or path, on a interval $[0, T]$ ) can be approximated further, as is often done in the asymptotic analysis of integrals. More precisely, a family of probability measures $\mathbb{P}_{\epsilon}$ on a polish space $\mathcal{X}$ is said to satisfy an LD principle [19] with rate function $I$ if, for all Borel measurable set $E \subset \mathcal{X}$

$$
\begin{aligned}
-\inf _{x \in E^{\circ}} I(x) & \leq \liminf _{\epsilon \rightarrow 0} \epsilon \log \left(\mathbb{P}_{\epsilon}(E)\right) \\
& \leq \limsup _{\epsilon \rightarrow 0} \epsilon \log \left(\mathbb{P}_{\epsilon}(E)\right) \leq-\inf _{x \in \bar{E}} I(x) .
\end{aligned}
$$

The reason to work with a liminf and limsup is mainly technical, and often we can interpret (9) simply as $\mathbb{P}_{\epsilon}(E) \approx$ $\exp \left\{-\inf _{x \in E} I(x) / \epsilon\right\}$. That is, every path $x$ toward the rare event $E$ has a certain "cost" $I(x)$, and the LDP tells us that, as $\epsilon \rightarrow 0$, the path with the smallest cost yields the largest probability and is therefore the most likely way for the event to happen. For further background, and other engineering applications of LD we refer to [42]; for an introduction of LD theory aimed at physicists, see [43].

This section is based on the Freidlin-Wentzell theory ( $\mathrm{F}-$ $\mathrm{W}$, [19]), which is concerned with large deviation principles for the paths of a stochastic process. Thanks to [19, Th. 5.6.7], the power injections process $X^{\epsilon}$ satisfies a sample path LD principle (SPLDP) over $C_{\mu}([0, T])=\{g:[0, T] \rightarrow$ $\mathbb{R}^{m}: g$ is continuous and $\left.g(0)=\mu\right\}$, with good rate function

$$
\mathcal{I}_{\text {pow }}(g)=\sum_{i=1}^{m} \mathcal{I}_{\text {pow }, i}\left(g_{i}\right)
$$

Here, $g=\left(g_{1}, \ldots g_{m}\right)$ and $\mathcal{I}_{\text {pow }, i}$ is the good rate function for the SPLDP associated with the process $X_{i}^{\epsilon}(t), i=1, \ldots, m$, and it is given by

$$
\mathcal{I}_{\text {pow }, i}\left(g_{i}\right)=\left\{\begin{array}{cl}
\frac{1}{2} \int_{0}^{T}\left(\frac{g_{i}^{\prime}-b_{i}\left(g_{i}\right)}{l_{i}\left(g_{i}\right)}\right)^{2} d t, & \text { if } g_{i} \in H_{\mu_{i}}^{1}(\mathbb{R}) \\
\infty, & \text { if } g_{i} \notin H_{\mu_{i}}^{1}(\mathbb{R})
\end{array} .\right.
$$

Here, $H_{\mu}^{1}\left(\mathbb{R}^{m}\right):=\left\{g:[0, T] \rightarrow \mathbb{R}^{m}: g(t)=\mu+\int_{0}^{t} \phi(s) d s\right.$, $\left.\phi \in L_{2}([0, T])\right\}$ is the space of absolutely continuous functions with value $\mu$ at 0 and which possess a square integrable derivative. Next, we apply a very useful tool in LD theory, known as the contraction principle, which allows to map LD principles from one space to another. Thanks to the contraction principle, [19, Th. 4.2.1], and (5), the current process $Y^{\epsilon}$ satisfies an SPLDP with good rate function

$$
\mathcal{I}_{\text {cur }}(f)=\inf _{\substack{g \in H_{\mu}^{1}: \\ y+C g=f}} \mathcal{I}_{\text {pow }}(g) .
$$

Thanks to Lemma II.1, the matrix $C$ has linear independent columns. Therefore, its Moore-Penrose inverse has an explicit formula $C^{+}=\left(C^{\top} C\right)^{-1} C^{\top}$ and it is a left inverse of $C$. Thus, for $f \in y+C\left(H_{\mu}^{1}\left(\mathbb{R}^{m}\right)\right) \subset H_{\nu}^{1}\left(\mathbb{R}^{L}\right)$, the equation $y+C g=$ $f$ has unique solution $g=C^{+}(f-y)$, yielding

$\mathcal{I}_{\text {cur }}(f)=\left\{\begin{array}{ll}\mathcal{I}_{\text {pow }}\left(C^{+}(f-y)\right), & \text { if } f \in y+C\left(H_{\mu}^{1}\left(\mathbb{R}^{m}\right)\right) . \\ \infty, & \text { otherwise }\end{array}\right.$.

For the current overload event, we then have that

$$
\begin{aligned}
& \limsup _{\epsilon \rightarrow 0} \epsilon \log \mathbb{P}\left(\left\|Y^{\epsilon}\right\| \geq 1\right)=-\mathcal{I}_{\text {cur }}^{*} \\
& \mathcal{I}_{\text {cur }}^{*}=\inf _{\substack{f \in y+C H_{\mu}^{1} \\
\|f\| \geq 1}} \mathcal{I}_{\text {cur }}(f)=\inf _{\substack{g \in H_{\mu}^{1}: \\
\|y+C g\| \geq 1}} \mathcal{I}_{\text {pow }}(g)
\end{aligned}
$$

with $\mathcal{I}_{\text {cur }}^{*}$ the decay rate for the current overload event. ${ }^{3}$

\section{B. Capacity Region Based on Current Overload}

Equation (13) yields the following approximation for the current overload probability for small $\epsilon$

$$
\mathbb{P}\left(\left\|Y^{\epsilon}\right\|_{\infty} \geq 1\right) \approx e^{-\mathcal{I}_{\text {cur }}^{*}(\bar{\mu}) / \epsilon} .
$$

We use the above-mentioned approximation to define the capacity region for the power grid, based on the constraint that the probability of current overflow must not exceed $p$, where $p>0$ is a small predefined threshold

$$
\tilde{\mathcal{R}}_{\epsilon, p}^{\text {(cur) }}:=\left\{\bar{\mu} \in \mathbb{R}^{N}: \mathcal{I}_{\text {cur }}^{*}(\bar{\mu}) \geq-\epsilon \log (p)\right\} .
$$

In the remainder of this section, we shed light on structural properties and computational aspects of this capacity region. Our first result shows that the capacity region is convex with respect to the deterministic power injections.

Lemma III.1: $\tilde{\mathcal{R}}_{\epsilon, p}^{(\text {cur })}$ is convex in the deterministic power injections vector $\mu_{D}$.

Lemma III.1 is important as convexity enables the set of allowable deterministic injections to be incorporated as a constraint in OPF problems (see, for example, [26]). For the special case where power injections are modeled as an OU process, we show in Section III-C that the capacity region $\tilde{\mathcal{R}}_{\epsilon, p}^{(\text {cur })}$ itself is convex. Letting

$$
\psi_{\ell}=\inf _{\substack{f \in H_{\nu}^{1}: \\\left\|f_{\ell}\right\|_{\infty} \geq 1}} \mathcal{I}_{\text {cur }}(f)=\inf _{\substack{g \in H_{\mu}^{1}: \\\left\|y_{\ell}+C_{\ell} g\right\|_{\infty} \geq 1}} \mathcal{I}_{\text {pow }}(g)
$$

with $C_{\ell}$ being the $\ell$ th row of matrix $C$, we note that $\mathcal{I}_{\text {cur }}^{*}=$ $\min _{\ell \in \mathcal{L}^{\prime}} \psi_{\ell}$, where $\mathcal{L}^{\prime}:=\left\{\ell \in \mathcal{L}: C_{\ell} \neq 0\right\}{ }^{4}$

In other words, the decay rate for a current overload in the network is the minimum of the decay rates corresponding to the overload of each line. Decay rates, together with (15), provide an analytical tool to rank transmission lines in terms of their vulnerability [44]. The next lemma shows that the current overload on any link most likely occurs at the end time.

\footnotetext{
${ }^{3}$ Note that $f \in H_{\nu}^{1} \backslash\left(y+C H_{\mu}^{1}\right)$ implies $\mathcal{I}_{\text {cur }}(f)=\infty$, thus $\mathcal{I}_{\text {cur }}^{*}=$ $\inf _{f \in y+C H_{\mu}^{1}:\|f\| \geq 1} \mathcal{I}_{\text {cur }}(f)=\inf _{f \in H_{\nu}^{1}:\|f\| \geq 1} \mathcal{I}_{\text {cur }}(f)$

${ }^{4}$ Note that if $C_{\ell}=0$, then $Y_{\ell}^{\epsilon}(t)=Y_{\ell}^{\epsilon}(0)=y_{\ell}$ is constant and $\left|y_{\ell}\right|=$

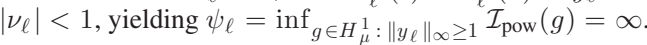


Lemma III.2: $\forall \ell \in \mathcal{L}^{\prime}, \psi_{\ell}=\inf _{g \in H_{\mu}^{1}:\left|y_{\ell}+C_{\ell} g(T)\right|=1} \mathcal{I}_{\text {pow }}(g)$.

For $a \neq \nu_{\ell}$, define

$$
\psi_{\ell}^{(a)}=\inf _{f \in y+C H_{\mu}^{1}: f_{\ell}(T)=a} \mathcal{I}_{\text {pow }}(f)
$$

so that $\psi_{\ell}=\psi_{\ell}^{(1)} \wedge \psi_{\ell}^{(-1)}=\min \psi_{\ell}^{(1)}, \psi_{\ell}^{(-1)}$ and

$$
\mathcal{I}_{\text {cur }}^{*}=\min _{\ell \in \mathcal{L}^{\prime}} \psi_{\ell}^{(1)} \wedge \psi_{\ell}^{(-1)} .
$$

Equation (18) allows us to rewrite the capacity region as

$$
\tilde{\mathcal{R}}_{\epsilon, p}^{\text {(cur) }}=\bigcap_{\ell \in \mathcal{L}^{\prime}, a \in\{-1,1\}}\left\{\bar{\mu} \in \mathbb{R}^{N}: \psi_{\ell}^{(a)} \geq-\epsilon \log (p)\right\} .
$$

Thus, obtaining the capacity region $\tilde{\mathcal{R}}_{\epsilon, p}^{(\text {cur })}$ hinges on computing $\psi_{\ell}^{(a)}$, which by definition is the solution of (17). To solve this variational problem with boundary constraints, one can for instance use the Euler-Lagrange equation (see also our discussion in Section V). For simple diffusion models, this approach can be used to obtain the optimal path and $\psi_{\ell}^{(a)}$ in closed form, leading to an explicit characterization of the capacity region $\tilde{\mathcal{R}}_{\epsilon, p}^{\text {(cur) }}$. Next, we illustrate this for the case where the power injections are modeled as an OU process.

\section{Explicit Computations for OU process}

In this section, we suppose that the power injections $X^{\epsilon}(t)$ follow a multivariate OU process, which is the most tractable example of an SDE and is, in particular, a Gaussian process. ${ }^{5}$ Such a process is of the form

$$
d X^{\epsilon}(t)=D\left(\mu-X^{\epsilon}(t)\right) d t+\sqrt{\epsilon} L d W(t)
$$

i.e., the functions $b(\cdot)$ and $L(\cdot)$ in the $\operatorname{SDE}(8)$ are $b(x)=D(\mu-$ $x)$ and $L(x)=L$, where $D=\operatorname{diag}\left(\left\{\gamma_{i}\right\}\right), L=\operatorname{diag}\left(\left\{l_{i}\right\}\right)$, and $\gamma_{i}, l_{i}>0$ for all $i=1, \ldots, m$. For this model, the capacity region can be expressed in closed form, as shown in the next Proposition. ${ }^{6}$

Proposition III.1: If $X^{\epsilon}(t)$ is defined by (20), then

$\tilde{\mathcal{R}}_{\epsilon, p}^{\text {(cur) }}=\bigcap_{\ell \in \mathcal{L}^{\prime}}\left\{\bar{\mu} \in \mathbb{R}^{N}:\left|\nu_{\ell}\right| \leq 1-\sqrt{\epsilon \log (1 / p) C_{\ell} M_{T} C_{\ell}^{\top}}\right\}$.

In the particular case $D=\gamma I$, (9) simplifies to

$$
\tilde{\mathcal{R}}_{\epsilon, p}^{\text {(cur) }}=\bigcap_{\ell \in \mathcal{L}^{\prime}}\left\{\bar{\mu} \in \mathbb{R}^{N}:\left|\nu_{\ell}\right| \leq 1-\beta_{\ell}\right\} .
$$

Here, $M_{t}=L^{2} D^{-1}\left(I-e^{-2 D t}\right) e^{D(t-T)}$ and

$$
\beta_{\ell}:=\sqrt{\frac{\left(1-e^{-2 \gamma T}\right) \epsilon \log (1 / p) \sigma_{\ell}^{2}}{\gamma}}, \quad \sigma_{\ell}^{2}:=C_{\ell} L^{2} C_{\ell}^{\top} .
$$

We make the following remarks regarding Proposition III.1.

\footnotetext{
${ }^{5}$ The gaussianity assumption for wind power is debatable. While consistent with atmospheric physics [26] and recent wind park statistics [45], [46], different models are preferred for different timescales [47].

${ }^{6}$ Note that our framework allows to extend Proposition III.1 to mixtures of OUs, providing flexibility to the modeler while keeping the benefits of closedform expressions.
}

1) $\tilde{\mathcal{R}}_{\epsilon, p}^{\text {(cur) }}$ is a closed convex set; in particular, it is a polyhedron in $\mathbb{R}^{N}$. We note that this property enables us to incorporate the capacity region in OPF problems.

2) $\beta_{\ell}$ is a strictly decreasing function of $\gamma$, implying that $\tilde{\mathcal{R}}_{\epsilon, p}^{(\text {cur })}$ shrinks as $\gamma$ becomes smaller. This is intuitive, since for small values of $\gamma$, the OU process will revert to its long-term mean $\mu$ with less force.

3) The longer the time $T$ between two control instants, the greater the probability that the fluctuations in the power injections will result in an overload, yielding a smaller $\tilde{\mathcal{R}}_{\epsilon, p}^{\text {(cur) }}$.

4) The expression for $\tilde{\mathcal{R}}_{\epsilon, p}^{\text {(cur) encloses in a single formula }}$ dependence on the initial condition $\nu$, on the window length $T$, and on the topology of the network, the physical properties of the transmission lines and the evolution of the stochastic power injections, encoded in the matrices $C, L$, and $D$.

\section{Capacity Regions Characterization Based on TEMPERATURE OVERLOAD}

Since temperature responds gradually to current, a current overload of a short duration does not necessarily imply an overload in temperature. By explicitly capturing the transient relationship between temperature and current, we can enlarge the conservative capacity region obtained in Section III. In the following, we first provide an $\mathrm{LD}$ principle for the temperature overload event $\mathbb{P}\left(\left\|\Theta^{\epsilon, \tau}\right\|_{\infty} \geq 1\right)$. Then, we define the temperature-overload-based capacity region and prove a convexity result for it, analogous to the result in Section III.

However, due to the nonlocal in time relationship between current and temperature, the decay rate for the temperature process is hard to compute explicitly. As a result, the capacity region cannot be expressed in closed form for even the simplest diffusion models. To address this issue, we develop two approximations of the capacity region: the first is an inner bound, whereas the second is based on a first order Taylor expansion of the decay rate around $\tau=0$. These approximations have the following appealing properties, which make them amenable to application in OPF formulations. First, both approximations are supersets of the current-based capacity region $\tilde{\mathcal{R}}_{\epsilon, p}^{\text {(cur) }}$. Second, they have the same computational complexity as $\tilde{\mathcal{R}}_{\epsilon, p}^{\text {(cur) }}$. Third, for the special case where the stochastic power injections are modeled by an OU process, both regions can be expressed in closed form (Sections IV-D and IV-E). Finally, both approximations are convex over the deterministic power injections.

\section{A. Capacity Region Based on Temperature Overload}

Thanks to the relationship (6), the contraction principle yields that $\Theta_{\epsilon, \tau}$ satisfies an SPLDP with good rate function

$$
\mathcal{I}_{\text {tmp }, \tau}(h)=\inf _{\substack{f \in H_{\nu}^{1}: \\ \xi_{\tau}(f)=h}} \mathcal{I}_{\text {cur }}(f)=\inf _{\substack{f \in y+C H_{\mu}^{1}: \\ \xi_{\tau}(f)=h}} \mathcal{I}_{\text {cur }}(f) .
$$


For the temperature overload event, we thus have

$$
\begin{aligned}
& \limsup _{\epsilon \rightarrow 0} \epsilon \log \mathbb{P}\left(\left\|\Theta^{\epsilon, \tau}\right\|_{\infty} \geq 1\right) \leq-\mathcal{I}_{\text {tmp }, \tau}^{*} \\
& \mathcal{I}_{\text {tmp }, \tau}^{*}=\inf _{\substack{h \in \xi_{\tau}\left(H_{\nu}^{1}\right) \\
\|h\| \geq 1}} \mathcal{I}_{\text {tmp }, \tau}(h)
\end{aligned}
$$

where $\mathcal{I}_{\text {tmp }, \tau}^{*}$ is the temperature decay rate. Letting, for $\ell \in \mathcal{L}^{\prime}$

$$
\omega_{\ell}=\inf _{\substack{h \in \xi_{\tau}\left(H_{\nu}^{1}\right): \\\left\|h_{\ell}\right\|_{\infty} \geq 1}} \mathcal{I}_{\text {tmp }, \tau}(h)=\inf _{\substack{g \in H_{\mu}^{1}: \\\left\|\xi_{\tau_{\ell}}\left(y_{\ell}+C_{\ell} g\right)\right\|_{\infty} \geq 1}} \mathcal{I}_{\text {pow }}(g)
$$

we see that the decay rate for the temperature is $\mathcal{I}_{\mathrm{tmp}, \tau}^{*}=$ $\min _{\ell \in \mathcal{L}^{\prime}} \omega_{\ell}$. Note that $\omega_{\ell}$ and $\mathcal{I}_{\text {tmp }, \tau}^{*}$ depend on $\bar{\mu}, \tau$, and $T$. As before, (23) yields the following approximation for the rare event probability, for small $\epsilon$

$$
\mathbb{P}\left(\left\|\Theta^{\epsilon, \tau}\right\|_{\infty} \geq 1\right) \approx e^{-\mathcal{I}_{\text {tmp }, \tau}^{*}(\bar{\mu}) / \epsilon} .
$$

This leads to the following definition of the capacity region

$$
\begin{aligned}
\tilde{\mathcal{R}}_{\epsilon, p}^{(\mathrm{tmp}, \tau)}: & =\left\{\bar{\mu} \in \mathbb{R}^{N}: \mathcal{I}_{\mathrm{tmp}, \tau}^{*}(\bar{\mu}) \geq-\epsilon \log (p)\right\} \\
& =\bigcap_{l \in \mathcal{L}^{\prime}}\left\{\bar{\mu} \in \mathbb{R}^{N}: \omega_{\ell}(\bar{\mu}) \geq-\epsilon \log (p)\right\} .
\end{aligned}
$$

We have the following convexity result.

Lemma IV.1: $\tilde{\mathcal{R}}_{\epsilon, p}^{(\mathrm{tmp}, \tau)}$ is convex in the deterministic power injections vector $\mu_{D}$.

The variational problem for the temperature overload (24) is difficult to solve in general, and numerics can also prove to be challenging. Motivated by this difficulty, in the next section, we develop approximations for the temperature decay rate, and the corresponding capacity regions, by reducing (24) to the easier problem (17).

\section{B. Inner Bound for the Capacity Region}

In this section, we develop an inner bound for the capacity region $\tilde{\mathcal{R}}_{\epsilon, p}^{(\mathrm{tmp}, \tau)}$, which is larger than the capacity region $\tilde{\mathcal{R}}_{\epsilon, p}^{\text {(cur) }}$ based on current overload, and thus captures some of the benefits of incorporating temperature dynamics. Define

$$
\mathcal{I}_{\mathrm{tmp}, \tau}^{(L B)}:=\min _{\ell \in \mathcal{L}^{\prime}} \psi_{\ell}^{\left(\alpha_{\ell}\right)} \wedge \psi_{\ell}^{\left(-\alpha_{\ell}\right)} .
$$

The next lemma shows that $\mathcal{I}_{\mathrm{tmp}, \tau}^{(L B)}$ is a lower bound for the temperature decay rate, i.e., $\mathcal{I}_{\mathrm{tmp}, \tau}^{*} \geq \mathcal{I}_{\mathrm{tmp}, \tau}^{(L B)}$.

Lemma IV.2: For all $\ell \in \mathcal{L}^{\prime}$, we have $\omega_{\ell} \geq \psi_{\ell}^{\left(\alpha_{\ell}\right)} \wedge \psi_{\ell}^{\left(-\alpha_{\ell}\right)}$, where $\alpha_{\ell}=\sqrt{\frac{1-\nu_{\ell}^{2} e^{-T / \tau} \ell}{1-e^{-T / \tau} \ell}}$.

The capacity region based on the lower bound $\mathcal{I}_{\mathrm{tmp}, \tau}^{(L B)}$ is

$$
\tilde{\mathcal{R}}_{\epsilon, p}^{(\mathrm{tmp}, \tau, L B)}:=\left\{\bar{\mu} \in \mathbb{R}^{N}: \mathcal{I}_{\mathrm{tmp}, \tau}^{(L B)}(\tau, \bar{\mu}) \geq-\epsilon \log (p)\right\} .
$$

The following proposition states that the capacity region based on the lower bound, while being an inner approximation of the actual temperature-based region, is less conservative than the current-based capacity constraint.

Proposition IV.1: $\mathcal{I}_{\text {cur }}^{*} \leq \mathcal{I}_{\text {tmp }, \tau}^{(L B)} \leq \mathcal{I}_{\text {tmp }, \tau}^{*}$ and $\tilde{\mathcal{R}}_{\epsilon, p}^{(\text {cur })} \subseteq$ $\tilde{\mathcal{R}}_{\epsilon, p}^{(\mathrm{tmp}, \tau, L B)} \subseteq \tilde{\mathcal{R}}_{\epsilon, p}^{(\mathrm{tmp}, \tau)}$ for all $\tau \geq 0$.
As a consequence, using $\tilde{\mathcal{R}}_{\epsilon, p}^{\text {(tmp }, \tau, L B)}$ over $\tilde{\mathcal{R}}_{\epsilon, p}^{\text {(cur) }}$ allows for larger power injections values (i.e., less curtailment) while still bounding the probability of a temperature overload and without additional computational burden. Finally, we note that the inner bound satisfies the following convexity property.

Lemma IV.3: $\tilde{\mathcal{R}}_{\epsilon, p}^{(\mathrm{tmp}, \tau, L B)}$ is convex in the deterministic power injections vector $\mu_{D}$.

The proof goes along the same lines of the proofs of Lemmas III.1 and IV.1 and is therefore omitted.

\section{Taylor Approximation of the Decay Rate and Corresponding Capacity Regions}

In this section, we derive a heuristic approximation for the temperature decay rate

$$
\mathcal{I}_{\mathrm{tmp}, \tau}^{*}=\inf _{h \in \xi_{\tau}\left(y+C H_{\mu}^{1}\right),\|h\| \geq 1} \mathcal{I}_{\mathrm{tmp}, \tau}(h)
$$

based on a Taylor expansion around $\tau=0$. First, write the temperature rate function (22) as

$$
\mathcal{I}_{\mathrm{tmp}, \tau}(h)= \begin{cases}G(\tau, h), & \text { if } h \in \xi_{\tau}\left(y+C H_{\mu}^{1}\right) \\ \infty, & \text { otherwise }\end{cases}
$$

where $G(\tau, h)$ is defined explicitly in the appendix.

Taylor approximation 1: Let $f_{*}$ be the optimal current path to overflow. For small $\tau$, we will use the approximation

$$
\mathcal{I}_{\text {tmp }, \tau}^{*} \approx \mathcal{I}_{\text {tmp }, \tau}^{(T L)}:=\mathcal{I}_{\text {cur }}^{*}+\left.\tau \cdot \nabla_{\tau} G\left(\tau, f_{*}^{2}\right)\right|_{\tau=0} .
$$

If $\tau$ is of the form $\tau=\tau_{0}(1, \ldots, 1)^{\top}, \tau_{0}>0$, we obtain the closed-form expression

$$
\mathcal{I}_{\mathrm{tmp}, \tau}^{(T L)}=\mathcal{I}_{\mathrm{cur}}^{*}+\tau_{0} \Phi_{f_{*}}
$$

where

$$
\begin{aligned}
& \Phi_{f_{*}}:=\sum_{i=1}^{m}\left[K_{i}\left(f_{*}(T), f_{*}^{\prime}(T)\right)-K_{i}\left(f_{*}(0), f_{*}^{\prime}(0)\right)\right] \\
& K_{i}\left(f_{*}(t), f_{*}^{\prime}(t)\right):=\frac{1}{2}\left(\frac{C_{i}^{+} f_{*}^{\prime}(t)-b_{i}\left(C_{i}^{+}\left(f_{*}(t)-y\right)\right)}{l_{i}\left(C_{i}^{+}\left(f_{*}(t)-y\right)\right)}\right)^{2} .
\end{aligned}
$$

In particular, the approximation $\mathcal{I}_{\text {tmp }, \tau}^{(T L)}$ depends only on the current decay rate $\mathcal{I}_{\text {cur }}^{*}$ and on the values $f_{*}(0),\left(f_{*}\right)^{\prime}(0), f_{*}(T),\left(f_{*}\right)^{\prime}(T)$.

The heuristic is motivated by the formal Taylor expansion of $I_{t, \tau}^{*}$ around $\tau=0$, i.e., $\mathcal{I}_{t, 0}^{*}+\left.\tau \cdot \nabla_{\tau} I_{t, \tau}^{*}\right|_{\tau=0}+o(\tau)$. If $\tau=0$, the optimal temperature path to overflow is $h_{*}=\left(f_{*}\right)^{2}$, so $\mathcal{I}_{\text {tmp }, \tau}^{*}=\mathcal{I}_{\text {cur }^{2}}\left(h_{*}\right)=\mathcal{I}_{\text {cur }}\left(f_{*}\right)=\mathcal{I}_{\text {cur }}^{*}$, and the substitution of $\left.\nabla_{\tau} G\left(\tau, f_{*}^{2}\right)\right|_{\tau=0}$ for $\left.\nabla_{\tau} \mathcal{I}_{\text {tmp }, \tau}^{*}\right|_{\tau=0}$ is motivated by an infinitedimensional version of Danskin's theorem [48, Proposition 4.13]. To make this rigorous is quite challenging, as the feasible sets in our variational problem depend in a rather intricate way on $\tau$. The explicit calculations for the case $\tau=\tau_{0}(1, \ldots, 1)^{\top}$, $\tau_{0}>0$, are reported in the appendix.

Equation (30) provides an approximation of the temperature decay rate that depends only on the current decay rate and the 
corresponding optimal path, which are generally easier to obtain. The capacity region corresponding to the Taylor approximation is

$$
\tilde{\mathcal{R}}_{\epsilon, p}^{(\mathrm{tmp}, \tau, T L)}:=\left\{\bar{\mu} \in \mathbb{R}^{N}: \mathcal{I}_{\text {cur }}^{*}(\bar{\mu})+\tau_{0} \Phi_{f_{*}} \geq-\epsilon \log (p)\right\} .
$$

In Section IV-E, we will see that in the OU case, the inequality $\mathcal{I}_{\text {tmp }, \tau}^{(T L)} \geq \mathcal{I}_{\text {cur }}^{*}$ holds, and thus $\tilde{\mathcal{R}}_{\epsilon, p}^{(t, \tau, T L)} \supseteq \tilde{\mathcal{R}}_{\epsilon, p}^{(\text {cur })}$, confirming the intuition that the temperature-based approach is less conservative than the current-based one.

\section{Explicit Computations for OU: Lower Bound}

In this section, we assume that the power injection process $X^{\epsilon}(t)$ follows the OU process (20), and we explicitly compute the lower bound $\mathcal{I}_{\text {tmp. },}^{(L B)}$ and the corresponding capacity region $\tilde{\mathcal{R}}_{\epsilon, p}^{(\operatorname{tmp}, \tau, L B)}$.

Proposition IV.2: If $X^{\epsilon}(t)$ is defined by (20), then

$$
\begin{aligned}
\mathcal{I}_{\mathrm{tmp}, \tau}^{(L B)} & =\min _{\ell \in \mathcal{L}^{\prime}} \frac{\left(\alpha_{\ell}-\left|\nu_{\ell}\right|\right)^{2}}{C_{\ell} M_{T} C_{\ell}^{\top}} \\
\tilde{\mathcal{R}}_{\epsilon, p}^{(\mathrm{tmp}, \tau, L B)} & =\bigcap_{\ell \in \mathcal{L}^{\prime}}\left\{\bar{\mu} \in \mathbb{R}^{N}: \frac{\left(\alpha_{\ell}-\left|\nu_{\ell}\right|\right)^{2}}{C_{\ell} M_{T} C_{\ell}^{\top}} \geq-\epsilon \log (p)\right\}
\end{aligned}
$$

where

$$
\alpha_{\ell}=\sqrt{\frac{1-\nu_{\ell}^{2} e^{-T / \tau_{\ell}}}{1-e^{-T / \tau_{\ell}}}}, \quad M_{t}=L^{2} D^{-1}\left(I-e^{-2 D t}\right) e^{D(t-T)} .
$$

In the particular case $D=\gamma I$, we have

$$
\begin{aligned}
& \tilde{\mathcal{R}}_{\epsilon, p}^{(\mathrm{tmp}, \tau, L B)}:=\bigcap_{\ell \in \mathcal{L}^{\prime}}\left\{\bar{\mu} \in \mathbb{R}^{N}:\left|\nu_{\ell}\right| \leq \delta_{\ell}\right\} \\
& \delta_{\ell}=\sqrt{1-\eta_{\ell}^{2} e^{-T / \tau_{\ell}}\left(1-e^{-T / \tau_{\ell}}\right)}-\eta_{\ell}\left(1-e^{-T / \tau_{\ell}}\right) \\
& \eta_{\ell}:=\sqrt{\frac{\epsilon \log (1 / p) \sigma_{\ell}^{2}\left(1-e^{-2 \gamma T}\right)}{\gamma}}<1, \sigma_{\ell}^{2}=C_{\ell} L^{2} C_{\ell}^{\top} .
\end{aligned}
$$

If $D=\gamma I$, we see from Proposition IV.2 that $\tilde{\mathcal{R}}_{\epsilon, p}^{(\operatorname{tmp}, \tau, L B)}$ is a convex polyhedron in $\mathbb{R}^{N}$, as in the case of the current region, and is in particular a scaled version of the polyhedron $\tilde{\mathcal{R}}_{\epsilon, p}^{\text {(cur) }}$. Moreover, $\delta_{\ell} \in\left(1-\eta_{\ell}, 1\right), \delta_{\ell} \stackrel{\tau \rightarrow \infty}{\longrightarrow} 1$ and $\delta_{\ell} \stackrel{\tau \rightarrow 0}{\longrightarrow} \eta_{\ell}$. This means that as $\tau$ increases, the capacity region (33) gets closer to the larger region $\left\{\bar{\mu} \in \mathbb{R}^{N}:\|\nu\|_{\infty}<1\right\}$, which is the stability region for a deterministic system. On the other hand, as $\tau \rightarrow 0$, the region in (33) boils down to the smaller current-based capacity region given in (9).

\section{E. Explicit Computations for OU: Taylor Approximation}

In this section, we consider again the OU process $X^{(\epsilon)}(20)$ in the particular case $D=\gamma I$, and we develop the capacity regions based on the Taylor approximation 1 .

$$
\begin{gathered}
\text { Proposition IV.3: For } \tau=\tau_{0}(1, \ldots, 1)^{\top} \text {, we have } \\
\mathcal{I}_{\text {tmp }, \tau}^{(T L)}=\left(1+2 \tau_{0} \gamma\right) \mathcal{I}_{\text {cur }}^{*}(\bar{\mu})=\left(1+2 \tau_{0} \gamma\right) \min _{\ell \in \mathcal{L}^{\prime}} \frac{\left(1-\left|\nu_{\ell}\right|\right)^{2}}{C_{\ell} M_{T} C_{\ell}^{\top}} \\
\tilde{\mathcal{R}}_{\epsilon, p}^{(\mathrm{mp}, \tau, T L)}=\bigcap_{\ell \in \mathcal{L}^{\prime}}\left\{\bar{\mu} \in \mathbb{R}^{N}:\left|\nu_{\ell}\right| \leq 1-\eta_{\ell} / \sqrt{1+2 \tau_{0} \gamma}\right\} .
\end{gathered}
$$

It is clear that $\tilde{\mathcal{R}}_{\epsilon, p}^{\left(t, t, \tau_{0} L\right)}$ is a convex polyhedron, as it was the case for the current region $\tilde{\mathcal{R}}_{\epsilon, p}^{\text {(cur) }}$ and the lower bound region $\tilde{\mathcal{R}}_{\epsilon, p}^{\left(t, \tau_{0}, L B\right)}$. Moreover, since $1+2 \tau_{0} \gamma>0$, we see that $\tilde{\mathcal{R}}_{\epsilon, p}^{(\mathrm{tmp}, \tau, T L)} \supseteq \tilde{\mathcal{R}}_{\epsilon, p}^{(\mathrm{cur})}$, and in particular, $\tilde{\mathcal{R}}_{\epsilon, p}^{(\mathrm{tmp}, \tau, T L)}$ is a rescaled version of $\tilde{\mathcal{R}}_{\epsilon, p}^{\text {(cur) }}$. Recall that this was also the case for the lower bound capacity region: the difference is that while the lower bound holds for every $\tau>0$, the approximation $\mathcal{I}_{\text {tmp }, \tau}^{(T L)}$ is good only for small $\tau_{0}$. In general, $\tilde{\mathcal{R}}_{\epsilon, p}^{(\mathrm{tmp}, \tau, T L)}$ and $\tilde{\mathcal{R}}_{\epsilon, p}^{(\mathrm{tmp}, \tau, L B)}$ are not subsets of each other.

\section{NUMERICS}

In order to compute the temperature decay rate $\mathcal{I}_{\text {tmp }, \tau}^{*}$, one has to solve the variational problem in (25), which is computationally harder than the one for the current in (17), due to the integral mapping in (7).

On the other hand, the theory we presented enables us to reduce the computation of the decay rates $\mathcal{I}_{\text {tmp }, \tau}^{(L B)}$ and $\mathcal{I}_{\text {tmp }, \tau}^{(T L)}$ to the easier variational problem for $\mathcal{I}_{\text {cur }}^{*}$, capturing the benefits of incorporating the temporal dynamics between current and temperature without additional cost. Variational problems, such as (17), which are based on F-W theory, are well studied in the literature, and when closed-form expression are not available, efficient numerical algorithms have been developed [50].

In the following sections, we apply our theory to derive the capacity regions for two IEEE test cases, and we quantify the capacity gains achieved by $\tilde{\mathcal{R}}_{\epsilon, p}^{(\operatorname{tmp}, \tau, L B)}, \tilde{\mathcal{R}}_{\epsilon, p}^{(\mathrm{tmp}, \tau, T L)}$ over $\tilde{\mathcal{R}}_{\epsilon, p}^{\text {(cur) }}$ assuming an OU model for power injections. The code used to produce Figs. 1 and 2 is available at github.com/TommasoNesti/Temperature-Overloads.

Scalability: Thanks to the analytic characterization of capacity regions for the OU model, our approach is fully scalable and can effortlessly be applied to larger power networks, as there is virtually no computational burden in computing $\tilde{\mathcal{R}}_{\epsilon, p}^{\text {(cur) }}$ and, therefore, all the other capacity regions. For a detailed analysis on computational costs for solving (17) for a general SDE, the interested reader is referred to $[50, \mathrm{Sec} .3 .3]$.

\section{A. IEEE-14 Test Network}

In this section, we develop capacity regions for the IEEE-14 test network, corresponding to the test case case 14 in [51].

The grid consists of 14 nodes and 20 lines, and the original test case has constant deterministic power injections $P_{D} \in \mathbb{R}^{14}$, expressed in the per-unit system. We replace two of the deterministic injections (nodes 2 and 13) by OU processes with long-term mean equal to the original deterministic power injection, and we assume that we control the injections at nodes 6 and 9. The test case reports the parameters $P_{D}, w_{i j}$, and 


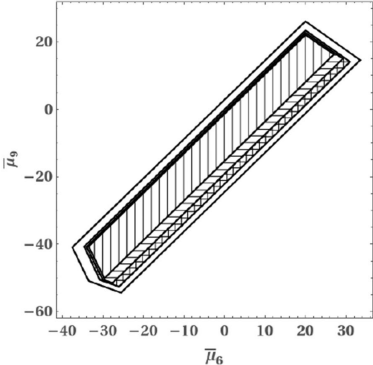

(a)

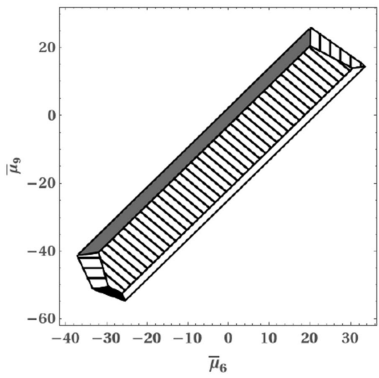

(c)

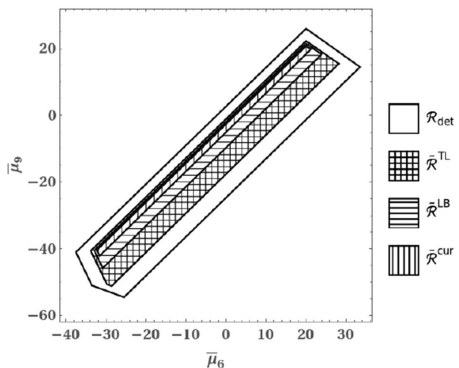

(b)
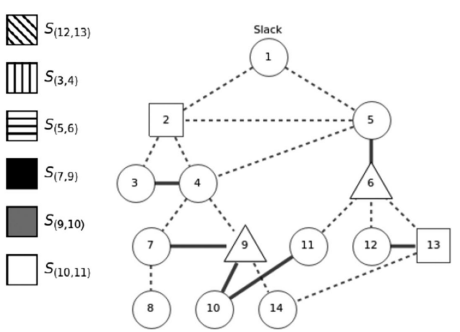

(d)
Fig. 1. (a) and (b) Capacity regions for the IEEE 14-bus network, depicted using different mesh styles, for two different target probabilities. (c) Subdivision of $\mathcal{R}_{\text {det }}$ according to which lines are the most vulnerable, as in (35). (d) IEEE-14 topology. Stochastic and (deterministic) controllable nodes are represented with square and triangular vertexes, respectively. The six solid lines are the most vulnerable ones.

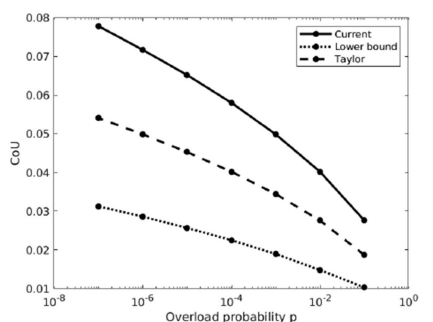

(a)

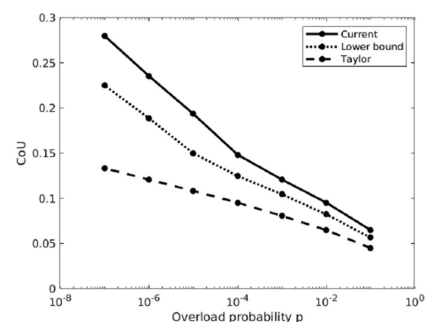

(b)
Fig. 2. $\operatorname{CoU}^{(\mathcal{R})}$ for different overload probabilities $p$ and time intervals $T, \mathcal{R} \in\left\{\tilde{\mathcal{R}}_{\epsilon, p}^{\text {(cur) }}, \tilde{\mathcal{R}}_{\epsilon, p}^{(\mathrm{tmp}, \tau, L B)}, \tilde{\mathcal{R}}_{\epsilon, p}^{(\mathrm{tmp}, \tau, T L)}\right\}$. (a) $T=1 / 4(15 \mathrm{~min}) . q(T)=$ 0.018. (b) $T=1$ (60 $\mathrm{min}), q(T)=0.04$.

$\widetilde{C}$, but does not include line limits, which we define as follows. For each line $\ell$, we set the maximum permissible current $I_{\ell, \max }=K\left|I_{\ell, \text { nom }}\right|, I_{\ell, \text { nom }}$ being the nominal current in line $\ell$ obtained from $P_{D}$ via the dc power flow equation, and $K=1.5$. We set $T=1, \gamma_{i}=1, l_{i}^{2}=10, \epsilon=0.25$, and $\tau=0.5$.

We compute two-dimensional capacity regions, which correspond to the amount of power that can be injected at the controllable sources so that the probability of overload in $[0, T]$ is sufficiently small. The current-based capacity region is

$$
\begin{aligned}
\tilde{\mathcal{R}}_{\epsilon, p}^{\text {(cur })}= & \left\{\left(\bar{\mu}_{6}, \bar{\mu}_{9}\right) \in \mathbb{R}^{2} \mid, \bar{\mu}=\left(P_{D, 2}, \ldots, P_{D, 5}, \bar{\mu}_{6}, P_{D, 7},\right.\right. \\
& \left.\left.P_{D, 8}, \bar{\mu}_{9}, P_{D, 10}, \ldots, P_{D, 14}\right), \mathcal{I}_{\text {cur }}(\bar{\mu}) \geq-\epsilon \log (p)\right\}
\end{aligned}
$$

and the other regions are defined similarly.

In Fig. 1(a) and (b), the two-dimensional capacity regions $\tilde{\mathcal{R}}_{\epsilon, p}^{(\text {cur })}, \tilde{\mathcal{R}}_{\epsilon, p}^{(\mathrm{tmp}, \tau, L B)}$, and $\tilde{\mathcal{R}}_{\epsilon, p}^{(\mathrm{tmp}, \tau, T L)}$ (denoted as $\tilde{\mathcal{R}}^{(\text {cur })}, \tilde{\mathcal{R}}^{(\mathrm{LB})}$, and $\tilde{\mathcal{R}}^{(\mathrm{TL})}$ in the legend) are shown for two different target probabilities $p$, together with the region corresponding to a deterministic system

$$
\mathcal{R}_{\text {det }}=\left\{\left(\mu_{1}, \mu_{2}\right) \in \mathbb{R}^{2}|| \nu_{\ell} \mid \leq 1 \quad \forall \ell=1,2,3\right\} .
$$

In particular, Fig. 1(b) shows that for $p=10^{-7}$, the lower bound region $\tilde{\mathcal{R}}_{\epsilon, p}^{(\mathrm{tmp}, \tau, L B)}$ is more than two times bigger than $\tilde{\mathcal{R}}_{\epsilon, p}^{(\text {cur })}$, and the Taylor region $\tilde{\mathcal{R}}_{\epsilon, p}^{(\mathrm{tmp}, \tau, T L)}$ is approximately two times bigger than $\tilde{\mathcal{R}}_{\epsilon, p}^{(\operatorname{tmp}, \tau, L B)}$. This result suggests that for small target probabilities, the temperature-based approach yields a significative capacity gain.

Another application of the proposed methodology is the identification of the most vulnerable parts of the grid. For a given value of $\bar{\mu}$, let $\ell^{*}(\bar{\mu}):=\operatorname{argmin}_{\ell \in \mathcal{L}^{\prime}} \psi_{\ell}^{(1)}(\bar{\mu}) \wedge \psi_{\ell}^{(-1)}(\bar{\mu})$ denote the line with the highest chance of overloading (18), and for a line $k \in \mathcal{L}^{\prime}$, define

$$
S_{k}:=\left\{\left(\bar{\mu}_{6}, \bar{\mu}_{9}\right) \in \mathbb{R}^{2} \mid\|\nu\| \leq 1, \ell^{*}(\bar{\mu})=k\right\} \subset \mathcal{R}_{\operatorname{det}} .
$$

The region $S_{k} \subset \mathcal{R}_{\text {det }}$ characterizes the controllable power injections such that in the event of large fluctuations of stochastic power injections, line $k$ is the most likely line to overload. The $S_{k}$-s partition $\mathcal{R}_{\text {det }}$ in several subregions, as shown in Fig. 1(c). Such characterization can help detecting the most vulnerable components of the grid: in this case, line $(12,13)$, corresponding to the biggest subregion in Fig. 1(c). Finally, Fig. 1(d) shows the topology of the network.

\section{B. IEEE-118 Test Network}

In this section, we perform a case study on a larger system, corresponding to the test case c118swf.m [52]. The system has 118 nodes, 210 lines, and 52 generators, 11 of which are modeled as wind units (indexed by $j_{1}, \ldots, j_{11}$ ). In order to simulate a more heavily loaded system, we define $I_{\max }$ to be equal to $50 \%$ of the line limits provided in the test case.

For our study, we first solve a dc OPF [53], which is an optimization problem determining the generation schedule that minimizes the total system generation cost while satisfying demand/supply balance and network physical constraints, under the assumptions of the dc approximations. Let $\bar{\mu} \in \mathbb{R}^{118}$ be the resulting optimal net power injections vector.

Next, we model the 11 wind generators as OU processes, using the hour as the unit for temporal quantities. The parameter $\mu_{k}$ of generator $j_{k}$ is set to be equal to $\bar{\mu}_{j_{k}}$, which is interpreted as the nominal power injection of generator $j_{k}$.

The parameters $\epsilon, D=\gamma I, L=\operatorname{diag}\left(\left\{l_{i}\right\}\right)$, and $T$ are calibrated in such a way that the standard deviation of each $\mathrm{OU}$ process at the end time $T$ matches realistic values for wind power forecasting error (expressed as a fraction of the wind plant installed capacity) over different control periods

$$
\operatorname{std}_{j_{k}}(T)=\sqrt{\frac{\epsilon l_{k}^{2}}{2 \gamma}\left(1-e^{-2 T \gamma}\right)}=q(T) \cdot \mu_{j_{k}}^{(\text {installed })}
$$

Given $T$, we set $q=q(T), \gamma=1, \epsilon=1$, and solve (36) for $l_{k}$. The values for $q(T)$, shown in Fig. 2(a) and (b), are taken from [54], and correspond to the root-mean-squared forecast 
error obtained applying a persistence forecast to ERCOT wind data. Note that this setting can capture renewable generators with different installed capacities. The overload probabilities are chosen in the range $\left[10^{-7}, 10^{-1}\right]$, and $\tau=0.5 \mathrm{~h}$.

To quantify the capacity gain achieved by the different regions, for each choice of the parameters, we solve three distinct dc OPFs, each incorporating a different capacity region $\mathcal{R}$ in the constraints. Note that since the capacity regions are convex polytopes, solving these OPFs has the same computational cost as solving the deterministic one.

Next, we compare the total system costs, which is the value of the objective function at optimality, to the cost obtained by solving the deterministic OPF (that is, the one incorporating $\mathcal{R}_{\text {det }}$ in the constraints), by means of the Cost of Uncertainty $(\mathrm{CoU})$ metric

$$
\operatorname{CoU}^{(\mathcal{R})}(q, p)=\frac{\operatorname{cost}^{\mathcal{R}}(q, p)-\operatorname{cost}^{\mathrm{det}}}{\operatorname{cost}^{\mathrm{det}}} \geq 0
$$

defined as the relative increase in system costs when uncertaintyaware reliability constraints are considered. Fig. 2 reports $\operatorname{CoU}(q, p)$ for various values of $q$ and $p$.

We see that enforcing constraints on line currents results in higher system costs than the ones achieved by using temperature-based constraints, consistently across different probability levels and time intervals. The gain is more pronounced over shorter intervals, capturing the intuition that current overloads are permissible for short periods, and for smaller probabilities: for instance, CoU drops from $8 \%$ to $3 \%$ when $\tilde{\mathcal{R}}_{\epsilon, p}^{(\mathrm{tmp}, \tau, L B)}$ is used over $\tilde{\mathcal{R}}_{\epsilon, p}^{(\mathrm{cur})}$, for $T=1 / 4$ and $p=10^{-7}$.

\section{CONCLUding REMARKS}

We employed LD theory to develop tractable capacity regions for power grids with variable power injections, modeled as small-noise diffusion processes, assuming currents behave according to the dc power flow equations. These capacity regions define the set of initial power injections such that the probability of a current/temperature overload in a given interval is very small, and can be used as computationally tractable chanceconstraints in OPF formulations. Incorporating the transient relationship between line temperature and line current leads to enlarged capacity regions. While this enlarged region is difficult to compute, we develop tractable approximations that improve upon the capacity region defined by the conservative current overload constraint.

A natural (and possibly straightforward) follow-up to this work would be to consider other linearizations of the ac power flow equations (for example, a first-order Taylor expansion around a nominal operating point). Extensions to more general classes of power injection processes present another interesting avenue for future work, as does the complementary task of fitting a suitable SDE model based on empirical data of renewable generation.

Finally, we note that the potential of our LD results goes beyond the development of capacity regions. Our results can be used to speed up more detailed simulations, as in [9]. In addition, the ranking of transmission lines according to their overload probability makes our techniques applicable to identify the most vulnerable parts of the network [44].

\section{APPENDIX}

Proof of Lemma II.1: Since $w_{\ell} \neq 0, I_{\ell, \max } \neq 0$ for all $\ell=1, \ldots, L$, the matrices $W$ and $\Lambda$ are nonsingular. Following [25], we see that $\operatorname{rank} \breve{B}=N$, and Lemma 2.2 in [55] guarantees that $\operatorname{rank} A=N$ and $\operatorname{Ker}(A)=\operatorname{Span}\left((1, \ldots, 1)^{T}\right)$. Since $W$ is nonsingular, $\operatorname{rank}(W A \breve{B})=\operatorname{rank}(A \breve{B})$, and $\operatorname{rank}(A \breve{B}) \leq \min (\operatorname{rank}(A), \operatorname{rank}(\breve{B}))=N$. On the other hand, if $x \in \operatorname{Ker}(A \breve{B})$ then $A \breve{B} x=0 \Longleftrightarrow \breve{B} x \in \operatorname{Ker}(A)=$ $\operatorname{Span}\left((1, \ldots, 1)^{T}\right) \Longleftrightarrow \breve{B} x=0 \Longleftrightarrow x \in \operatorname{Ker}(\breve{B})$, where in the second implication, we used that the first component of $\breve{B} x$ is 0 . Therefore, $\operatorname{dim} \operatorname{Ker}(A \breve{B})=\operatorname{dim} \operatorname{Ker}(\breve{B})=1$, yielding $\operatorname{rank}(A \breve{B})=N+1-\operatorname{dim} \operatorname{Ker}(A \breve{B})=N$. Since $\Lambda$ is nonsingular, the matrix $\bar{C}$ must have $N$ linear independent columns. But its first column is zero (since the first column of $\breve{B}$ is zero), therefore the columns from 2 to $m \leq N$ of $\bar{C}$ are linearly independent, i.e. the matrix $C$ has full rank $m$.

Proof of Lemma III.1: First notice that a vector $\left(\mu, \mu_{D}\right)$ such that $\|\nu\|=\left\|C \mu+C_{D} \mu_{D}\right\|<1$ belongs to the capacity

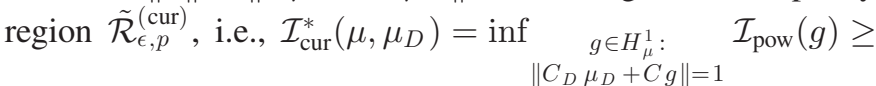
$\epsilon \log (1 / p)$, if and only if the following implication holds:

$$
\exists g \in H_{\mu}^{1} \text { s.t. } \mathcal{I}_{\text {pow }}(g)<\epsilon \log (1 / p) \Rightarrow\left\|C_{D} \mu_{D}+C g\right\|<1 \text {. }
$$

Consider two admissible vectors $\left(\mu, \mu_{D}\right),\left(\mu, \tilde{\mu}_{D}\right) \in \tilde{\mathcal{R}}_{\epsilon, p}^{\text {(cur) }}$, and let $\lambda \in[0,1]$. We want to show that $\left(\mu, \lambda \mu_{D}+(1-\lambda)\right)$ $\tilde{\mu}_{D} \in \tilde{\mathcal{R}}_{\epsilon, p}^{(\text {cur })}$. To this end, take $g \in H_{\mu}^{1}$ to be such that $\mathcal{I}_{\text {pow }}(g)<$ $\epsilon \log (1 / p)$, and let us write $\| \lambda C_{D} \mu_{D}+(1-\lambda) C_{D} \tilde{\mu}_{D}+$ $C g\|=\| \lambda\left(C_{D} \mu_{D}+C g\right)+(1-\lambda)\left(C_{D} \tilde{\mu}_{D}+C g\right)\|\leq \lambda\|$ $\left(C_{D} \mu_{D}+C g\right)\|+(1-\lambda)\|\left(C_{D} \tilde{\mu}_{D}+C g\right) \|<\lambda+(1-\lambda)=$ 1 , where we used property $(A .1)$ and the fact that $\left(\mu, \mu_{D}\right)$ and $\left(\mu, \tilde{\mu}_{D}\right)$ are admissible. Therefore, $\lambda \mu_{D}+(1-\lambda) \tilde{\mu}_{D}$ is admissible (notice that the aforementioned calculation implies in particular that $\| C \mu+C_{D}\left(\lambda \mu_{D}+(1-\lambda) \tilde{\mu}_{D} \|<1\right)$.

Proof of Lemma III.2: Define $S_{1}=\left\{g \in H_{\mu}^{1}\right.$ : $\left.\left\|C_{\ell} g(t)+y_{\ell}\right\|_{\infty} \geq 1\right\}, S_{2}=\left\{g \in H_{\mu}^{1}:\left|C_{\ell} g(T)+y_{\ell}\right|=1\right\}$. We have to prove that inf $\operatorname{ins}_{g} \mathcal{I}_{\text {pow }}(g)=\inf _{g \in S_{2}} \mathcal{I}_{\text {pow }}(g)$. Since $S_{2} \subset S_{1}$, it follows that $\inf _{g \in S_{1}} \mathcal{I}_{\text {pow }}(g) \leq \inf _{g \in S_{2}} \mathcal{I}_{\text {pow }}(g)$. To prove the reverse inequality, we show that for any $g \in S_{1}$, there exists $\tilde{g} \in S_{2}$ such that $\mathcal{I}_{\text {pow }}(\tilde{g}) \leq \mathcal{I}_{\text {pow }}(g)$. Pick $g \in S_{1}$. Let $t^{\prime} \in[0, T]$ be the first time such that $\left|y_{\ell}+C_{\ell} g\left(t^{\prime}\right)\right|=1$. Clearly $t^{\prime}>0$, since $\left|y_{\ell}+C_{\ell} g(0)\right|=\left|\nu_{\ell}\right|<1$. If $t^{\prime}=T$, we may take $\tilde{g}(t)=g(t)$. If $t^{\prime}<T$, define $\tilde{g}(t)$ by time-shifting $g(t)$ to the right as follows:

$$
\tilde{g}(t)=\left\{\begin{array}{ll}
\mu, & \text { for } 0 \leq t<T-t^{\prime} \\
g\left(t-T+t^{\prime}\right), & \text { for } T-t^{\prime} \leq t \leq T
\end{array} .\right.
$$

It is easy to check that $\tilde{g} \in S_{2}$, and that $\mathcal{I}_{\text {pow }}(\tilde{g}) \leq \mathcal{I}_{\text {pow }}(g)$, because the path $\tilde{g}$ incurs no cost up to time $T-t^{\prime}$. Indeed, since in the interval $\left[0, T-t^{\prime}\right] \tilde{g}$ is constantly equal to $\mu$, we have $b(\tilde{g}(t))=b(\mu)=0$ and $\tilde{g}^{\prime}(t)=0$, yielding 
$\int_{0}^{T-t^{\prime}}\left(\frac{g_{i}^{\prime}-b_{i}\left(g_{i}\right)}{l_{i}\left(g_{i}\right)}\right)^{2} d t=0$ and thus

$$
\begin{aligned}
\mathcal{I}_{\text {pow }}(\tilde{g}) & =\int_{T-t^{\prime}}^{T}\left(\frac{\tilde{g}_{i}^{\prime}-b_{i}\left(\tilde{g}_{i}\right)}{l_{i}\left(\tilde{g}_{i}\right)}\right)^{2} \\
d t & =\int_{0}^{t^{\prime}}\left(\frac{g_{i}^{\prime}-b_{i}\left(g_{i}\right)}{l_{i}\left(g_{i}\right)}\right)^{2} d t \leq \mathcal{I}_{\text {pow }}(g) .
\end{aligned}
$$

Lemma VI.1: The function $a \rightarrow \psi_{\ell}^{(a)}$ is nondecreasing for $a>\nu_{\ell}$ and nonincreasing for $a<\nu_{\ell}$.

Proof: First suppose $a \geq \tilde{a}>\nu_{\ell} \geq 0$. The case $a \leq \tilde{a}<$ $\nu_{\ell} \leq 0$ is analogous. We want to show that for all $f \in y+C H_{\mu}^{1}$ such that $f_{\ell}(T)=a$, there exist a $\tilde{f} \in y+C H_{\mu}^{1}$ with $\tilde{f}_{\ell}(T)=$ $\tilde{a}$ and $\mathcal{I}_{\text {cur }}(\tilde{f}) \leq \mathcal{I}_{\text {cur }}(f)$. Since $f_{\ell}(0)=\nu_{l}<\tilde{a} \leq a$ and $f$ is continuous, there exist a $t^{\prime} \in(0, T)$ such that $f\left(t^{\prime}\right)=\tilde{a}$. Define $\tilde{f}(t)$ as follows:

$$
\tilde{f}(t)=\left\{\begin{array}{ll}
\nu, & \text { for } 0 \leq t<T-t^{\prime} \\
f\left(t-T+t^{\prime}\right), & \text { for } T-t^{\prime} \leq t \leq T
\end{array} .\right.
$$

It is easy to check that $\tilde{f} \in y+C H_{\mu}^{1}, \tilde{f}_{\ell}(T)=\tilde{a}$, and $\mathcal{I}_{\text {cur }}(\tilde{f}) \leq$ $\mathcal{I}_{\text {cur }}(f)$. The proof that $\psi_{\ell}^{(a)}$ is nonincreasing for $a<\nu_{\ell}$ goes along the same lines.

Proof of Proposition III.1: Following the methods in [9], for $\ell \in \mathcal{L}^{\prime}=\left\{\ell \in \mathcal{L}: C_{\ell} \neq 0\right\}$ it can be shown that

$$
\psi_{\ell}^{(a)}=\frac{\left(a-\nu_{\ell}\right)^{2}}{C_{\ell} M_{T} C_{\ell}^{\top}}
$$

where $M_{t}=L^{2} D^{-1}\left(I-e^{-2 D t}\right) e^{D(t-T)}$. The corresponding optimal paths for power injections and currents leading to the overload of line $\ell$ are

$$
\begin{aligned}
X^{(\ell)}(t) & =\left(a-\nu_{\ell}\right) \frac{M_{t} C_{\ell}^{\top}}{C_{\ell} M_{T} C_{\ell}^{\top}}+\mu \in \mathbb{R}^{m} \\
Y^{(\ell)}(t) & =C X^{(\ell)}(t)+y \in \mathbb{R}^{L} .
\end{aligned}
$$

It follows easily that

$$
\mathcal{I}_{\text {cur }}^{*}(\bar{\mu})=\min _{\ell \in \mathcal{L}^{\prime}} \frac{\left(1-\left|\nu_{\ell}\right|\right)^{2}}{C_{\ell} M_{T} C_{\ell}^{\top}} .
$$

A straightforward calculation yields the desired result.

Proof of Lemma IV.1: First notice that a vector $\left(\mu, \mu_{D}\right)$ such that $\|\nu\|=\left\|C \mu+C_{D} \mu_{D}\right\|<1$ is admissible if and only if the following implication holds:

$$
\exists g \in H_{\mu}^{1} \text { s.t. } \mathcal{I}_{\text {pow }}(g)<\epsilon \log (1 / p) \Rightarrow\left\|h^{g, \mu, \mu_{D}}\right\|_{\infty}<1
$$

where

$$
\begin{aligned}
& h_{\ell}^{g, \mu, \mu_{D}}(t):=\xi_{\tau}(y+C g)=\left(y_{\ell}+C_{\ell} \mu\right)^{2} e^{-t / \tau} \\
& +\frac{1}{\tau} \int_{0}^{t} e^{-(t-s) / \tau}\left(y_{\ell}+C_{\ell} g(s)\right)^{2} d s, \quad y=C_{D} \mu_{D} .
\end{aligned}
$$

For all $\ell \in \mathcal{L}$ and for all $t \in[0, T], h_{\ell}^{g, \mu, \mu_{D}}(t)$ is nonnegative and convex in $\mu_{D}$. Using the property (A.5), the rest of the proof goes along the lines of the proof of Lemma III.1.
Proof of Lemma IV.2: The proof follows easily from the observation that the event $\left\|\Theta_{\ell}^{\epsilon, \tau}\right\|_{\infty} \geq 1$ implies the event $\left\|Y_{\ell}^{\epsilon}\right\|_{\infty} \geq \alpha_{\ell}$. Indeed, it is easy to check that if $\left|Y_{\ell}^{\epsilon}(t)\right|<\alpha_{\ell}$ for all $t \in[0, T]$, then it follows from (6) that $\Theta_{\ell}^{\epsilon, \tau}(t)<1$ for all $t \in[0,1]$. Thus, we have

$$
\begin{aligned}
\omega_{\ell} & =\lim _{\epsilon \downarrow 0}-\epsilon \log P\left(\left\|\Theta_{\ell}^{\epsilon, \tau}\right\|_{\infty} \geq 1\right) \geq \lim _{\epsilon \downarrow 0}-\epsilon \log P\left(\left\|Y_{\ell}^{\epsilon}\right\|_{\infty} \geq \alpha_{\ell}\right) \\
& =\inf _{g:\left\|y_{\ell}+C_{\ell} g\right\|_{\infty} \geq \alpha_{\ell}} \mathcal{I}_{\text {pow }}(g)=\inf _{g:\left|y_{\ell}+C_{\ell} g(T)\right|=\alpha_{\ell}} \mathcal{I}_{\text {pow }}(g) \\
& =\psi_{\ell}^{\left(\alpha_{\ell}\right)} \wedge \psi_{\ell}^{\left(-\alpha_{\ell}\right)} .
\end{aligned}
$$

Proof of Proposition IV.1: Thanks to Lemma IV.2, we see that $\mathcal{I}_{\mathrm{tmp}, \tau}^{(L B)}$ is a lower bound for the temperature decay rate, i.e., $\mathcal{I}_{\text {tmp }, \tau}^{*} \geq \mathcal{I}_{\text {tmp }, \tau}^{(L B)}$. Since $\alpha_{\ell}>1>\left|\nu_{\ell}\right| \quad \forall \ell$, Lemma VI.1 implies $\psi_{\ell}^{\left(\alpha_{\ell}\right)} \wedge \psi_{\ell}^{\left(-\alpha_{\ell}\right)} \geq \psi_{\ell}^{(1)} \wedge \psi_{\ell}^{(-1)}$, yielding $\mathcal{I}_{\text {tmp }, \tau}^{(L B)} \geq \mathcal{I}_{\text {cur }}^{*}$.

Proof of Proposition IV.2: Thanks to Lemma IV.2, we have $\mathcal{I}_{\text {tmp }, \tau}^{(L B)}=\min _{\ell \in \mathcal{L}^{\prime}} \psi_{\ell}^{\left(\alpha_{\ell}\right)} \wedge \psi_{\ell}^{\left(-\alpha_{\ell}\right)}$. From (A.2), we get $\psi_{\ell}^{\left(\alpha_{\ell}\right)}=$ $\frac{\left(\alpha_{\ell}-\nu_{\ell}\right)^{2}}{C_{\ell} M_{T} C_{\ell}^{T}}$ and thus $\psi_{\ell}^{\left(\alpha_{\ell}\right)} \wedge \psi_{\ell}^{\left(-\alpha_{\ell}\right)}=\psi_{\ell}^{\left(\operatorname{sign}\left(\nu_{\ell}\right) \alpha_{\ell}\right)}=\frac{\left(\alpha_{\ell}-\left|\nu_{\ell}\right|\right)^{2}}{C_{\ell} M_{T} C_{\ell}^{T}}$, yielding the expression for $\tilde{\mathcal{R}}_{\epsilon, p}^{(\mathrm{tmp}, \tau, L B)}$. In the case $D=\gamma I$, a straightforward calculation yields the result.

Proof of Proposition IV.3: In the case $D=\gamma I$, according to (A.3), the optimal current paths to overflow in line $\ell$ and the corresponding decay rates are

$$
\begin{aligned}
Y^{(\ell)}(t) & =\left(\operatorname{sign}\left(\nu_{\ell}\right)-\nu_{\ell}\right) \frac{\left(1-e^{-2 \gamma t}\right) e^{\gamma(t-T)}}{1-e^{2 \gamma T}} R^{(\ell)}+\nu \\
\psi_{\ell} & =\frac{\gamma}{1-e^{-2 \gamma T}} \frac{\left(1-\left|\nu_{\ell}\right|\right)^{2}}{\sigma_{\ell}^{2}}
\end{aligned}
$$

where $R^{(\ell)}:=\frac{C L^{2} C_{\ell}^{T}}{C_{\ell} L^{2} C_{\ell}^{T}} \in \mathbb{R}^{L}$ and $\sigma_{\ell}^{2}=C_{\ell} L^{2} C_{\ell}^{T}$. Take any $\ell^{*} \in \arg \min _{\ell \in \mathcal{L}^{\prime}} \psi_{\ell}$. Recall that $\ell^{*}$ depends on the initial condition $\bar{\mu}$, i.e., $\ell^{*}=\ell^{*}(\bar{\mu})$. Letting $S^{*}=\operatorname{sign}\left(\nu_{\ell^{*}}\right)-\nu_{\ell^{*}} \in$ $\mathbb{R}$ and $R^{*}=R^{\left(\ell^{*}\right)}$, the optimal current path to overflow is $f_{*}(t)=Y^{\left(\ell^{*}\right)}(t)$ and in particular $f_{*}(0)=\nu, f_{*}(T)=S^{*} R^{*}+$ $\nu,\left(f_{*}\right)^{\prime}(0)=\frac{2 \gamma e^{\gamma T}}{1-e^{-2 \gamma T}} S^{*} R^{*}$, and $\left(f_{*}\right)^{\prime}(T)=\frac{\gamma\left(1+e^{-2 \gamma T}\right)}{1-e^{-2 \gamma T}} S^{*} R^{*}$. After a lengthy but straightforward calculation, which is reported in the extended preprint version [49], the formula for the Taylor approximation reads

$$
\mathcal{I}_{\text {tmp }, \tau}^{(T L)}(\bar{\mu})=\left(1+2 \tau_{0} \gamma\right) \mathcal{I}_{\text {cur }}^{*}(\bar{\mu}) .
$$

The capacity region defined by the Taylor approximation is

$$
\begin{aligned}
& \tilde{\mathcal{R}}_{\epsilon, p}^{(\mathrm{tmp}, \tau, T L)} \\
& =\bigcap_{\ell \in \mathcal{L}^{\prime}}\left\{\bar{\mu} \in \mathbb{R}^{N}: \frac{\gamma\left(1-\left|\nu_{\ell}\right|\right)^{2}}{\left(1-e^{-2 \gamma T}\right) \sigma_{\ell}^{2}}\left(1+2 \tau_{0} \gamma\right)>-\epsilon \log (p)\right\}
\end{aligned}
$$

which can be rewritten as

$$
\tilde{\mathcal{R}}_{\epsilon, p}^{(\mathrm{tmp}, \tau, T L)}=\bigcap_{\ell \in \mathcal{L}^{\prime}}\left\{\bar{\mu} \in \mathbb{R}^{N}:\left|\nu_{\ell}\right|<1-\eta_{\ell} / \sqrt{1+2 \tau_{0} \gamma}\right\} .
$$


Proof of (31): Since $\xi_{\tau}(f)=h$ if and only if $\tau h^{\prime}+h=f^{2}$, the temperature rate function reads

$$
\begin{aligned}
\mathcal{I}_{\text {tmp }, \tau}(h) & = \begin{cases}G(\tau, h), & \text { if } h \in \xi_{\tau}\left(y+C H_{\mu}^{1}\right) \\
\infty, & \text { otherwise }\end{cases} \\
G(\tau, h) & =\mathcal{I}_{\text {cur }^{2}}\left(\tau h^{\prime}+h\right)=\mathcal{I}_{\text {cur }}\left(f_{\tau h^{\prime}+h}\right) \\
& =\mathcal{I}_{\text {pow }}\left(C^{+}\left(f_{\tau h^{\prime}+h}-y\right)\right)
\end{aligned}
$$

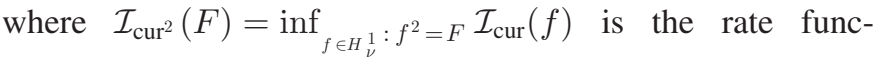
tion for the current-squared process $\left(Y^{\epsilon}(t)\right)^{2}$ and $f_{F}:=$ $\arg \min _{f \in H^{1}}: \mathcal{I}_{\text {cur }}(f)$. Note that $\mathcal{I}_{\text {cur }^{2}}(F)$ can be written as

$$
f^{2}=F
$$

$$
\mathcal{I}_{\text {cur }^{2}}(F)=\sum_{i=1}^{m} \int_{0}^{T} K_{i}\left(F(t), F^{\prime}(t)\right) d t
$$

$$
K_{i}\left(F(t), F^{\prime}(t)\right)=\frac{1}{2}\left[\frac{C_{i}^{+} f_{F}^{\prime}(t)-b_{i}\left(C_{i}^{+}\left(f_{F}(t)-y\right)\right)}{l_{i}\left(C_{i}^{+}\left(f_{F}(t)-y\right)\right)}\right]^{2} .
$$

The partial derivatives of the function

$$
\tau \rightarrow K_{i}\left(\left(\tau h^{\prime}+h\right),\left(\tau h^{\prime \prime}+h^{\prime}\right)\right)
$$

in $\tau=0$ read

$$
\begin{aligned}
& \left.\frac{\partial}{\partial \tau_{\ell}} K_{i}\left(\tau h^{\prime}+h, \tau h^{\prime \prime}+h^{\prime}\right)\right|_{\tau=0} \\
& =K_{i}^{(\ell)}\left(h, h^{\prime}\right) h_{\ell}^{\prime}+K_{i}^{(L+\ell)}\left(h, h^{\prime}\right) h_{\ell}^{\prime \prime}
\end{aligned}
$$

yielding

$$
\begin{aligned}
& \left.\sum_{\ell=1}^{L} \frac{\partial}{\partial \tau_{\ell}} K_{i}\left(\tau h^{\prime}+h, \tau h^{\prime \prime}+h^{\prime}\right)\right|_{\tau=0}=\frac{d}{d t} K_{i}\left(h, h^{\prime}\right) \\
& \left.\sum_{\ell=1}^{L} \frac{\partial}{\partial \tau_{\ell}} G(\tau, h)\right|_{\tau=0}=\left.\sum_{\ell=1}^{L} \frac{\partial}{\partial \tau_{\ell}} \mathcal{I}_{\operatorname{cur}^{2}}\left(\tau h^{\prime}+h\right)\right|_{\tau=0} \\
& =\left.\sum_{\ell=1}^{L} \sum_{i=1}^{m} \int_{0}^{T} \frac{\partial}{\partial \tau_{\ell}} K_{i}\left(\tau h^{\prime}+h, \tau h^{\prime \prime}+h^{\prime}\right)\right|_{\tau=0} \\
& =\sum_{i=1}^{m} \int_{0}^{T} \frac{d}{d t} K_{i}\left(h, h^{\prime}\right) d t=\sum_{i=1}^{m}\left[K_{i}\left(h(T), h^{\prime}(T)\right)\right. \\
& \left.-K_{i}\left(h(0), h^{\prime}(0)\right)\right]=: \Phi\left(f_{h}(0),\right. \\
& \left.f_{h}(T), f_{h^{\prime}}(0), f_{h^{\prime}}(T)\right)=: \Phi_{f_{h}} .
\end{aligned}
$$

If $\tau=\tau_{0}(1, \ldots, 1)^{T}, \tau_{0}>0$, we get $\left.\tau \cdot \nabla G(\tau, h)\right|_{\tau=0}=$ $\tau_{0} \Phi_{f_{h}}$. Finally, formula (30) follows by noticing that if $f_{*}$ is the optimal current path and $h_{*}=\left(f_{*}\right)^{2}$ then $f_{h_{*}}=f_{*}$.

\section{REFERENCES}

[1] Y. Yang, "Hybrid grids," Strategic Res. Innov. Position Paper 2, DNV GL, Oslo, Norway, 2015

[2] E. Ela, M. Milligan, and B. Kirby, "Operating reserves and variable generation," Nat. Renewable Energy Lab., Golden, CO, USA, Tech. Rep. TP-5500-51978, 2011.

[3] A. Mills and R. Wiser, "Implications of wide-area geographic diversity for short-term variability of solar power," Lawrence Berkeley Nat. Lab. Berkeley, CA, USA, Tech. Rep. LBNL-3884E, 2010.
[4] B. Hodge, S. Shedd, and A. Florita, "Examining the variability of wind power output in the regulation time frame," Nat. Renewable Energy Lab., Golden, CO, USA, Tech. Rep. CP-5500-55967, 2012.

[5] W. Fu and J. McCalley, "Risk based optimal power flow," in Proc. IEEE Porto Power Tech Conf., 2001, 6 pp.

[6] W. Wadman, G. Bloemhof, D. Crommelin, and J. Frank, "Probabilistic power flow simulation allowing temporary current overloading," in Proc. Int. Conf. Probabilistic Methods Appl. Power Syst., 2012, pp. 494-499.

[7] J. F. Shortle, "Efficient simulation of blackout probabilities using splitting," Int. J. Elect. Power Energy Syst., vol. 44, pp. 743-751, 2013.

[8] J. Bosman, J. Nair, and B. Zwart, "On the probability of current and temperature overloading in power grids: A large deviations approach," ACM SIGMETRICS Perform. Eval. Rev., vol. 42, pp. 33-35, 2014.

[9] W. S. Wadman, D. T. Crommelin, and B. P. Zwart, "A large-deviationbased splitting estimation of power flow reliability," ACM Trans. Model. Comput. Simul., vol. 26, 2016, Art. no. 23

[10] E. B. Iversen, J. M. Morales, J. K. Møller, and H. Madsen, "Short-term probabilistic forecasting of wind speed using stochastic differential equations," Int. J. Forecasting, vol. 32, pp. 981-990, 2016

[11] J. K. Møller, M. Zugno, and H. Madsen, "Probabilistic forecasts of wind power generation by stochastic differential equation models," J. Forecasting, vol. 35, pp. 189-205, 2016.

[12] E. B. Iversen, J. M. Morales, J. K. Møller, P.-J. Trombe, and H. Madsen, "Leveraging stochastic differential equations for probabilistic forecasting of wind power using a dynamic power curve," Wind Energy, vol. 20, pp. 33-44, 2017.

[13] K. Purchala, L. Meeus, D. Van Dommelen, and R. Belmans, "Usefulness of DC power flow for active power flow analysis," in Proc. IEEE Power Eng. Soc. Gen. Meeting, 2005, pp. 2457-2462.

[14] L. Powell, Power System Load Flow Analysis. New York, NY, USA: McGraw-Hill, 2004.

[15] B. Stott, J. Jardim, and O. Alsac, "DC power flow revisited," IEEE Trans. Power Syst., vol. 24, no. 3, pp. 1290-1300, Aug. 2009.

[16] S. Iwamoto and Y. Tamura, "A load flow calculation method for illconditioned power systems," IEEE Trans. Power App. Syst., vol. PAS-100, no. 4, pp. 1736-1743, Apr. 1981.

[17] J. Baillieul and C. I. Byrnes, "Geometric critical point analysis of lossless power system models," IEEE Trans. Circuits Syst., vol. CAS-29, no. 11, pp. 724-737, Nov. 1982.

[18] D. K. Molzahn, B. C. Lesieutre, and C. L. DeMarco, "A sufficient condition for power flow insolvability with applications to voltage stability margins," IEEE Trans. Power Syst., vol. 28, no. 3, pp. 2592-2601, Aug. 2013

[19] A. Dembo and O. Zeitouni, Large Deviations Techniques and Applications. Berlin, Germany: Springer, 1998.

[20] H. Wan, J. McCalley, and V. Vittal, "Increasing thermal rating by risk analysis," IEEE Trans. Power Syst., vol. 14, no. 3, pp. 815-828, Aug. 1999.

[21] U.S.-Canada Power System Outage Task Force, "Final report on the August 14, 2003 blackout in the United States and Canada," Washington, DC, USA, and Ottawa, Canada, 2004.

[22] Federal Energy Regulatory Commission and North American Electric Reliability Corporation, "Arizona-Southern California outages on September 8, 2011," Washington, DC and Atlanta, GA, USA, Tech. Rep., 2012.

[23] D. Phan and S. Ghosh, "Two-stage stochastic optimization for optimal power flow under renewable generation uncertainty," ACM Trans. Model. Comput. Simul., vol. 24, 2014, Art. no. 2.

[24] T. Summers, J. Warrington, M. Morari, and J. Lygeros, "Stochastic optimal power flow based on convex approximations of chance constraints," in Proc. Power Syst. Comput. Conf., 2014, pp. 1-7.

[25] D. Bienstock, J. Blanchet, and J. Li, "Stochastic models and control for electrical power line temperature," Energy Syst., vol. 7, pp. 173-192, 2013.

[26] D. Bienstock, M. Chertkov, and S. Harnett, "Chance-constrained optimal power flow: Risk-aware network control under uncertainty," SIAM Rev., vol. 56, pp. 461-495, 2014

[27] M. Vrakopoulou, K. Margellos, J. Lygeros, and G. Andersson, Probabilistic Guarantees for the N-1 Security of Systems With Wind Power Generation. New Delhi, India: Springer, 2013.

[28] S. Bolognani and F. Dörfler, "Fast scenario-based decision making in unbalanced distribution networks," in Proc. Power Syst. Comput. Conf., Jun. 2016, pp. 1-7.

[29] M. Vrakopoulou, M. Katsampani, K. Margellos, J. Lygeros, and G. Andersson, "Probabilistic security-constrained ac optimal power flow," in Proc. IEEE Grenoble Conf., Jun. 2013, pp. 1-6. 
[30] L. Roald and G. Andersson, "Chance-constrained ac optimal power flow: Reformulations and efficient algorithms," IEEE Trans. Power Syst., vol. 33, no. 3, pp. 2906-2918, May 2018.

[31] G. Calafiore and M. C. Campi, "Uncertain convex programs: Randomized solutions and confidence levels," Math. Program., vol. 102, pp. 25-46, 2005.

[32] A. Nemirovski and A. Shapiro, "Convex approximations of chance constrained programs,” SIAM J. Optim., vol. 17, pp. 969-996, 2006.

[33] N. Li, L. Chen, and S. Low, "Optimal demand response based on utility maximization in power networks," in Proc. IEEE Power Energy Soc. Gen. Meeting, 2011, pp. 1-8.

[34] S. Madaeni and R. Sioshansi, "The impacts of stochastic programming and demand response on wind integration," Energy Syst., vol. 4, pp. 109-124, 2013.

[35] J. Barton and D. Infield, "Energy storage and its use with intermittent renewable energy," IEEE Trans. Energy Convers., vol. 19, no. 2, pp. 441448, Jun. 2004.

[36] J. H. Kim and W. B. Powell, "Optimal energy commitments with storage and intermittent supply," Oper. Res., vol. 59, pp. 1347-1360, 2011.

[37] C. Weber, "Adequate intraday market design to enable the integration of wind energy into the European power systems," Energy Policy, vol. 38, pp. 3155-3163, 2010 .

[38] J. Nair, S. Adlakha, and A. Wierman, "Energy procurement strategies in the presence of intermittent sources," in Proc. ACM SIGMETRICS, 2014, pp. 85-97.

[39] J. Kersulis, I. Hiskens, M. Chertkov, S. Backhaus, and D. Bienstock, "Temperature-based instanton analysis: Identifying vulnerability in transmission networks," in Proc. IEEE Eindhoven PowerTech, Jun. 2015, pp. $1-6$.

[40] C. Coffrin, P. Van Hentenryck, and R. Bent, "Approximating line losses and apparent power in AC power flow linearizations," in Proc. IEEE Power Energy Soc. Gen. Meeting, 2012, pp. 1-8.

[41] H. Pender and W. Del Mar, Electrical Engineers' Handbook. Hoboken, NJ, USA: Wiley, 1949.

[42] J. A. Bucklew, Large Deviation Techniques in Decision, Simulation, and Estimation. New York, NY, USA: Wiley, 1990.

[43] H. Touchette, "The large deviation approach to statistical mechanics," Phys. Rep., vol. 478, pp. 1-69, 2009.

[44] T. Nesti, A. Zocca, and B. Zwart, "Emergent failures and cascades in power grids: A statistical physics perspective," Phys. Rev. Lett., vol. 120, 2018, Art. no. 258301.

[45] S. Kolumban, S. Kapodistria, and N. Nooraee, "Short and long-term wind turbine power output production," 2017, arXiv:1707.06497. [Online]. Available: https://arxiv.org/abs/1707.06497.

[46] J. Berg, A. Natarajan, J. Mann, and E. Patton, "Gaussian vs non-Gaussian turbulence: Impact on wind turbine loads," Wind Energy, vol. 19, pp. 19751989, 2016.

[47] P. Milan, M. Wächter, and J. Peinke, "Turbulent character of wind energy," Phys. Rev. Lett., vol. 110, Mar. 2013, Art. no. 138701.

[48] J. F. Bonnans and A. Shapiro, Perturbation Analysis Optimization Problems. Berlin, Germany: Springer, 2000.

[49] T. Nesti, J. Nair, and B. Zwart, "Temperature overloads in power grids under uncertainty: A large deviations approach," 2019, arXiv:1606.02986. [Online]. Available: https://arxiv.org/abs/1606.02986

[50] M. Heymann and E. Vanden-Eijnden, "The geometric minimum action method: A least action principle on the space of curves," Commun. Pure Appl. Math., vol. 61, pp. 1052-1117, 2008.

[51] R. D. Zimmerman, C. E. Murillo-Sánchez, and R. J. Thomas, "MATPOWER: Steady-state operations, planning, and analysis tools for power systems research and education," IEEE Trans. Power Syst., vol. 26, no. 1, pp. 12-19, Feb. 2011

[52] C. E. Murillo-Sánchez, R. D. Zimmerman, C. L. Anderson, and R. J. Thomas, "Secure planning and operations of systems with stochastic sources, energy storage, and active demand," IEEE Trans. Smart Grid, vol. 4, no. 4, pp. 2220-2229, Dec. 2013.
53] J. Sun and L. Tesfatsion, "DC optimal power flow formulation and solution using QuadProgJ," Econ. Working Papers, Iowa State Univ., Ames, IA, USA, 2010.

[54] B. Hodge and M. Milligan, "Wind power forecasting error distributions over multiple timescales," in Proc. IEEE Power Energy Soc. Gen. Meeting Jul. 2011, pp. 1-8.

[55] R. B. Bapat, Graphs and Matrices. Berlin, Germany: Springer, 2010

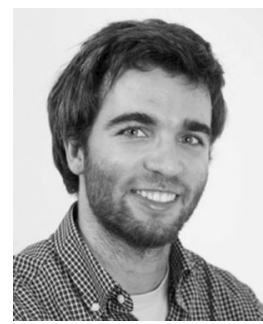

Tommaso Nesti received the B.Sc. and M.Sc. degrees in mathematics from the University of Pisa, Pisa, Italy, in 2015. He is currently working toward the Ph.D. degree in applied mathematics in the Stochastic Group, Centrum Wiskunde \& Informatica, Amsterdam, The Netherlands.

His research expertise is in the area of applied probability and numerical analysis. His current research focuses on problems arising in the study of energy systems under uncertainty, with a special interest for the analysis of rare events, such as cascading failures and blackouts, and for energy prices forecasting.

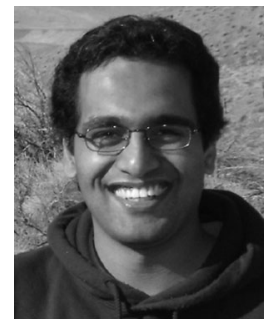

Jayakrishnan Nair received the B.Tech. and M.Tech. degrees in electrical engineering from the Indian Institute of Technology (IIT) Bombay, Mumbai, India, in 2007, and the Ph.D. degree in electrical engineering from the California Institute of Technology, Pasadena, CA, USA, in 2012.

He has held the postdoctoral positions with the California Institute of Technology and Centrum Wiskunde \& Informatica. He is currently an Assistant Professor in electrical engineering at IIT Bombay. His research focuses on modeling, performance evaluation and design issues in queueing systems and communication networks. His recent interests include game-theoretic interactions in queueing systems and smart power grids.

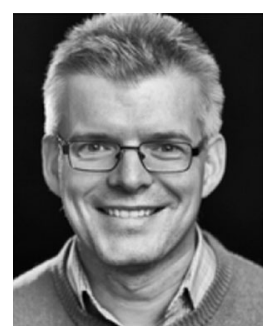

Bert Zwart (M'17) received the M.A. degree in econometrics from VU University of Amsterdam, the Netherlands, in 1997, and the Ph.D. degree in mathematics from TU Eindhoven, the Netherlands, in 2001.

$\mathrm{He}$ is currently the Leader of the Stochastics Group with the Centrum Wiskunde \& Informatica, Amsterdam, The Netherlands, and a Professor with the Eindhoven University of Technology, Eindhoven, The Netherlands. His research expertise is in applied probability and stochas tic networks. His work on power systems focuses on the applications of probabilistic methods to scheduling and reliability issues and rare events such as cascading failures and blackouts.

Prof. Zwart has been a Stochastic Models Area Editor for Operations Research, the flagship journal of his profession, from 2009-2017 and currently serves on the editorial boards of five journals. He was coorganizer of a special semester on the mathematics of energy systems that took place in Cambridge, U.K., Spring 2019. 